\title{
Molecular Mechanisms of Danhong Injection in Treatment of Pulmonary Fibrosis Based on Network Pharmacology and Experimental Validation
}

\section{Di Han}

Affiliated Hospital of Nanjing University of Chinese Medicine: Jiangsu Province Academy of Traditional Chinese Medicine

\section{Yong Xu}

Affiliated Hospital of Nanjing University of Chinese Medicine: Jiangsu Province Academy of Traditional Chinese Medicine

\section{Wenpan Peng}

Affiliated Hospital of Nanjing University of Chinese Medicine: Jiangsu Province Academy of Traditional Chinese Medicine

\section{Dan Wang}

Affiliated Hospital of Nanjing University of Chinese Medicine: Jiangsu Province Academy of Traditional Chinese Medicine

\section{Tongxing Huang}

Affiliated Hospital of Nanjing University of Chinese Medicine: Jiangsu Province Academy of Traditional Chinese Medicine

\section{Xianmei Zhou}

Affiliated Hospital of Nanjing University of Chinese Medicine: Jiangsu Province Academy of Traditional Chinese Medicine

\section{Jinjun Shan ( $\sim$ jshan@njucm.edu.cn )}

Jiangsu key laboratory of pediatric respiratory disease; Institute of Pediatrics https://orcid.org/00000001-8079-7950

\section{Research}

Keywords: Danhong injection, Pulmonary fibrosis, network pharmacology, Pi3k/Akt, apoptosis

Posted Date: June 11th, 2021

DOI: https://doi.org/10.21203/rs.3.rs-590776/v1

License: (c) (1) This work is licensed under a Creative Commons Attribution 4.0 International License. Read Full License 

1 Molecular mechanisms of Danhong injection in treatment of

2 Pulmonary Fibrosis based on network pharmacology and

3 experimental validation

4

Di Han ${ }^{1,2 \dagger}$, Yong $\mathrm{Xu}^{1,2 \dagger}$, Wenpan Peng ${ }^{1,2}$, Dan Wang ${ }^{1,2}$, Tongxing Huang ${ }^{1,2}$, Xianmei Zhou $^{1,2^{*}}$ and Jinjun Shan ${ }^{3,4^{*}}$

*Correspondence: zhouxianmeijs@aliyun.com; jshan@njucm.edu.cn 'Di Han and Yong Xu contributed equally to this work

${ }^{1}$ Affiliated Hospital of Nanjing University of Chinese Medicine, Nanjing, 210023 China

Full list of author information is available at the end of the article

\section{Abstract}

Backgroud: Danhong injection has been proven to be reliable and practical in the clinical treatment of Pulmonary Fibrosis $(\mathrm{PF})$, whereas the mechanism of action is unclear. The aim of this study was to investigate the mechanism of action of Danhong injection in the treatment of PF through network pharmacology and experimental validation.

Methods: In this paper, multiple databases were utilized to capture the targets of Danhong injection and PF disease-related targets. The interaction network such as active ingredient-target was constructed and protein interaction analysis was performed via Cytoscape software. Bioprocess and signaling pathway analysis of the targets was carried out through DIVID database. Based on the analytical results, pharmacodynamic validation and mechanistic investigation were conducted in mice.

Results: In this study, 111 compounds and common targets for diseases were filtered, with key targets involving MDM2, IL-2, CCL5, AKT1, MMP9, CASP3, MMP2, etc. 
The KEGG and GO enrichment analysis identified 16 significantly different signaling pathways, encompassing bioprocesses such as cell proliferation, apoptosis, programmed death and immune response. Animal experiments demonstrated that Danhong injection could reduce bleomycin-induced PF in mice, and could decrease the mRNA of AKT1 and the protein expression of p-Pi3k and p-Akt, thereby reducing apoptosis in mouse lung tissues.

Conclusions: This study initially revealed that Danhong injection may inhibit apoptosis through the Pi3k/Akt signaling pathway and thus reduce PF in mice.

Keywords: Danhong injection; Pulmonary fibrosis; network pharmacology; Pi3k/Akt; apoptosis

\section{Backgroud}

Among chronic respiratory diseases, Pulmonary Fibrosis (PF) is another "killer" in addition to lung cancer, which severely affects the quality of life among patients and significantly increases the economic burden on families and society[1]. Previous studies have demonstrated that the survival period for patients diagnosed with PF is only 2-3 years, with a 5-year survival rate of less than $30 \%[2]$. Although PF is a rare disease, the morbidity and mortality rates are increasing every year with no effective drugs available[3].

PF is classified as 'consumptive lung disease' and 'Bi syndrome of Lung' in Chinese medicine, with 'Bi syndrone of Lung' predominating in the early stage and 'consumptive lung disease' developing in the late stage[4]. The disease pathogenesis is 
essential empty and out solid. The essential empty is a deficiency of the lung, spleen and kidney, while the out solid is a paralysis of the lung channels by phlegm, heat and stasis. It is recognizedthat stagnated blood is a constant factor throughout the development of PF, thus PF can be treated based on the stagnated blood[5]. Traditional Chinese medicine features multi-component, multi-target and multi-pathway, and is widely used for its unique advantages in the treatment of sophisticated diseases[6]. Danhong injection is composed of Radix Salviae and Carthami Flos. Radix Salviae is bitter and slightly cold in nature, while Carthami Flos is pungent and warm in nature. The two drugs complement each other and work together to activate blood circulation, remove blood stasis, open the veins and relax the ligaments. According to extensive clinical studies, Danhong injection can improve the overall clinical efficiency, improve lung function, and diminish inflammatory response[7].

In this study, a "herbal-active component-target-disease" network was constructed through network pharmacology and experimental validation to investigate the active components and potential targets of Danhong injection for the treatment of PF, and to validate them through animal experiments.

\section{Materials and Methods}

\section{Databases}

Traditional Chinese Medicine Systems Pharmacology Database and Analysis Pla tform (TCMSP, http://tcmspw.com/tcmsp.php), Traditional Chinese Medicines Int egrated Database (TCMID, http://119.3.41.228:8000/tcmid/), Similarity ensemble approach (SEA, http://sea.bkslab.org), SwissTargetPrediction databases (http://ww 
w.swisstargetprediction.ch), Universal Protein (UniProt, http://www.uniprot.org/), DAVID Bioinformatics Resources (https://david.ncifcrf.gov/), STRING databases (https://string-db.org), Online Mendelian Inheritance in Man (OMIM, http://www. omim.org/), GeneCards (http://www.genecards.org), Pharmmapper (http://www.lila b-ecust.cn/pharmmapper/), Comparative Toxicogenomics Database (CTD, http://w ww.genecards.org ), Therapeutic Target Database (TTD, http://bidd.nus.edu.sg/gro up/ttd/ttd.asp).

\section{Acquisition of potential targets}

The TCMSP, TCMID, SEA and SwissTargetPrediction databases were accessed for the active ingredients and potential targets of Salvia and Safflower. The OMIM, GeneCards, CTD and TTD databases were accessed for disease-related targets, with the search term "Pulmonary Fibrosis". The VennDiagram package in R was applied to obtain common targets by mapping drug potential targets to disease-related targets.

\section{Plotting network and PPI diagrams}

In this study, the files "Drug_Disease.txt", "network.txt" and "type.txt" were imported into the Cytoscape software. Cytoscape software was used to draw the network diagrams, and the common target information was imported into String Online Analysis Platform with the minimum confidence set to 0.9 for protein-protein interaction analysis, and the generated "string_interactions.tsv" file into Cytoscape software for topological analysis. The core targets with values greater than their mean values were 
selected based on degree and betweenness centrality.

\section{Enrichment analysis}

In order to describe and annotate the functions of the target genes and to explore the signaling pathways involved, the core targets screened in 1.4 were subjected to GO and KEGG enrichment analyses through the DAVID database for the selected species "Homo sapiens". The GO enrichment analysis consisted of Molecular Function (MF), Biological Process (BP) and Cellular Components (CC). The results of both GO and KEGG enrichment analyses were filtered with a corrected $\mathrm{P}$ value (FDR) $<0.05$.

\section{Experimental validation}

\section{Reagents and antibodies}

The primary antibodies used in this study included antibodies to p-Pi3k, Pi3k, p-Akt, Akt, and $\beta$-tubulin purchased from CST (Danvers, Massachusetts, U.S.). Danhong injection (Shandong Buchang Pharmaceutical) was purchased from Jiangsu Provincial Hospital of Traditional Chinese Medicine (Nanjing, China). Bleomycin sulphate was purchased from TCI (Tokyo, Japan).

\section{Animal models}

Fifty male C57/BL6 mice were randomly divided into a normal control group, a model group, a Danhong injection group and a positive drug group, with 10 mice in each group. The normal control group was left untreated, and the rest of the groups were treated 
with a single intratracheal dose of bleomycin at a concentration of $5 \mathrm{mg} / \mathrm{ml}$. On the following day after operation, the normal control and model control groups were given saline gavage, the positive drug group was given pirfenidone $300 \mathrm{ml} / \mathrm{kg} / \mathrm{d}$ by gavage, and the Danhong injection group was given Danhong injection $3 \mathrm{ml} / \mathrm{kg} / \mathrm{d}$ and $6 \mathrm{ml} / \mathrm{kg} / \mathrm{d}$ by intraperitoneal injection once a day for $21 \mathrm{~d}$. The body weight of the mice was weighed every 3 days to observe the changes in body weight. Three hours after the last dose, the mice were killed by cervical dissection and the lung tissues were stored at $80{ }^{\circ} \mathrm{C}$.

\section{Haematoxylin and eosin (H\&E) staining and Masson staining}

Lung tissues were fixed with $4 \%$ neutral-buffered formalin for $24 \mathrm{~h}$. After that the colon was eluted with gradient ethanol and then immersed in xylene for $1 \mathrm{~h}$. The paraffinembedded lung samples were sliced into 4- $\mu$ m-thick sections, stained with haematoxylin and eosin, imaged under a light microscope for routine histopathological examination.

After dewaxing, sectioned samples was stained with hematoxylin for $30 \mathrm{~s}$, hydrochloric acid to induce differentiation into blue, Masson compound staining solution for $2 \mathrm{~min}, 1 \%$ phosphomolybdate and bright green staining solution for $5 \mathrm{~min}$, respectively. The sections were washed 3 times with $0.2 \%$ acetic acid after each staining, then dehydrated with anhydrous alcohol, cleaned with xylene until transparent, and sealed tightly with neutral glue. Images were obtained at $20 \times$ the original magnification. 


\section{Immunohistochemistry (IHC)}

For immunohistochemical analysis, paraffin-embedded lung tissue sections were deparaffined and antigens removed. The following experimental steps were performed according to the instructions of the UltraSensitive ${ }^{\mathrm{TM}} \mathrm{sp}$ immunohistochemical staining kit (MAIXIN.BIO, Fuzhou, China). Sections were stained with DAB solution (MAIXIN.BIO) and then re-stained with hematoxylin. Collagen I and collagen III expression was quantified by Image $\mathrm{J}$.

\section{Lung function tests}

After anesthesia, the mice were fixed supine on the operating table. The trachea was exposed and a cannula was inserted into the trachea and secured with cotton thread. After transferring the mice to a body description platform, lung compliance and lung resistance were measured.

\section{RNA isolation and quantitative real-time PCR (qRT-PCR)}

Total RNA was extracted using Trizol reagent (Invitrogen; Carlsbad, CA) in accordance with the manufacturer's instructions. cDNA was obtained via reverse transcription, which was performed using the $5 \times$ All-in-One RT MasterMix (Applied Biological Materials Inc; Richmond, Canada). mRNA expression levels were analyzed via quantitative real-time PCR using EvaGreen 2× qPCR Master Mix (Applied Biological Materials Inc; Richmond Canada). Relative mRNA expression levels of target genes were determined by normalizing values to expression levels of GAPDH. Data analysis 
was conducted using the comparative $\Delta \Delta \mathrm{CT}$ method. The following primers were synthesized by Sangon Biotech (Shanghai, China). The primer sequence is shown in Table 1.

\section{Western Blotting (WB)}

The lung tissue was lysed in RIPA containing 1\% PMSF (Beyotime, Nanjing, China), and total protein levels were determined using the pierce BCA protein analysis kit (Thermo Scientific; Rockford, IL). Proteins (20 g/well) were separated via SDSpolyacrylamide gel electrophoresis and subsequently transferred to a PVDF membrane (Merck MILI; Darmstadt, Germany). Subsequently, the membrane was incubated with $5 \%$ skim milk in Tris-buffered saline containing $0.1 \%$ Tween 20 (TBST $25^{\circ} \mathrm{C}$ for $1 \mathrm{~h}$. Then, the membrane and primary antibody were incubated overnight at $4^{\circ} \mathrm{C}$. Proteins were detected by incubating with a rabbit or mouse $\operatorname{IgG}(\mathrm{H}+\mathrm{L})$ antibody labeled with a peroxidase enzyme (Plano, TX). Visualization of target bands was accomplished using a super ECL detection kit (Yeasen; Shanghai, China). Protein levels were standardized using $\beta$-tubulin

\section{Enzyme-linked immunosorbent assay (ELISA)}

Levels of Hydroxyproline、Caspase -3、Caspase-8、Caspase-9 within the mouse lung tissue were determined using ELISA kits (Nanjing Jiancheng Bioengineering Institute, China). Results are based on three experiments.

\section{TUNEL assay}


The detection procedure was performed according to the manufacturer's instructions, but with modifications. Deparaffinized 4- $\mu$ m-thick tissue sections were treated with 20 $\mu \mathrm{g} / \mathrm{ml}$ proteinase $\mathrm{K}$ for $15 \mathrm{~min}$ at room temperature. Subsequently, the TUNEL reagent containing dUTP and TdT (Roche Diagnostics) was added to each section, and incubated in a humidified chamber at $37^{\circ} \mathrm{C}$ for $60 \mathrm{~min}$. The sections were rinsed with PBS and the sections were incubated with $0.3 \%$ converter-peroxidase for $30 \mathrm{~min}$ at $37^{\circ} \mathrm{C}$, then the sections rinsed with PBS prior to the addition of DAB substrate $10 \mathrm{~min}$ at room temperature and color rendering was observed under the microscope for 3-8 min. After the color development with tap water, sections were rinsed for $15 \mathrm{~min}$ and hematoxylin staining was performed for $2 \mathrm{~min}$ at room temperature, and subsequent steps were performed as follows. At the room temperature, the sections rinsed with tap water and differentiated with hydrochloric acid alcohol for 3-8 sec, then the slices were immersed in $70 \%$ alcohol at $5 \mathrm{~min}, 80 \%$ alcohol at $5 \mathrm{~min}, 90 \%$ alcohol at $5 \mathrm{~min}$ and $100 \%$ alcohol at 5 min twice, soaked in xylene at 10 min twice, and sealed with neutral balsam. The sections were observed by light microscopy (magnification, 20x).

\section{Data analysis}

The ggplot2, ggpubr, Rmisc and ComplexHeatmap data packages in $\mathrm{R}$ language software were used for statistical analysis and visualization; one-way ANOVA was used for comparative analysis using Graphpad Prism software, expressed as mean \pm standard deviation $(\overline{\mathrm{x}} \pm \mathrm{s}) . \mathrm{p}<0.05$ indicates a statistically significant difference. 


\section{Results}

\section{Active ingredients and potential targets of Danhong injection}

Under the limited conditions of $\mathrm{OB} \geq 30 \%$ and $\mathrm{DL} \geq 0.18 \%[8]$, a total of 85 acti ve ingredients were obtained by searching the TCMSP database for the two he rbal medicines of Radix Salviae and Carthami Flos (Table 2), of which 65 wer e from Radix Salviae and 22 from Carthami Flos. luteolin and Baicalin were c ommon to both Salvia miltiorrhiza and Safflower. The highest bioavailability w as obtained from (2R)-3-(3,4-dihydroxyphenyl)-2-[(Z)-3-(3,4-dihydroxyphenyl)acry loyl]oxy-propionic acid of Radix Salviae. The compounds collected were de-wei ghted and finally screened for 84 active ingredients (Table.2). The OB is the $\mathrm{p}$ roportion of the administered drug that enters the body's circulation and describ es the percentage of the oral dose that is absorbed by the gastrointestinal tract and passes through the liver to reach the bloodstream in the body's circulation, while the DL refers to the similarity of the compounds to known drugs.

\section{Drug-disease intersection targets}

A total of 599 Salvia miltiorrhiza and safflower-related targets and 5311 PF-related targets were obtained through database screening. The potential targets of Salvia and Safflower were mapped to PF-related targets, and 111 common targets were obtained and made into a Venn diagram (Fig. 2).

\section{Anti-PF network diagram of the active ingredients of Danhong injection}

In the drug-target-disease network diagram, the blue nodes represent the active 
ingredient information, the purple nodes represent the signaling pathways, the red nodes represent the drug and the green nodes represent the target information. A topological analysis of the network diagram revealed that there were 120 nodes and 1306 connecting lines, with a network centralization of 0.597 , network heterogeneity of 1.006 and network density of 0.177 (Fig.3).

\section{Screening of the core targets of the active ingredient of Danhong injection against} PF

The number of nodes connected represents the degree value and the median centrality represents the core degree of the node in the disease, both of which are important indicators to describe the nodes. The 111 common targets obtained in 2.2 were imported into the String online analysis platform, PPI maps were created and topological analysis was performed, and nodes with degree and median centrality greater than their mean values were retained as core targets. The results showed that a total of 21 core targets were filtered (Fig.4).

\section{Anti-PF enrichment analysis of the active ingredients of Danhong injection}

GO enrichment analysis (BP: 20, CC: 2, MF: 12) showed that the effects of Danhong injection were related to cell proliferation and apoptosis, cell bioregulation, cell biosynthesis, nucleic acid metabolic processes and protein transcription and translation (Fig. 5a); KEGG results showed that a total of 16 signaling pathways were closely related to $\mathrm{PF}$, namely Pi3k/Akt signaling pathway, Chemokine signaling pathway, Thyroid hormone signaling pathway, Jak-STAT signaling pathway, GnRH signaling 
pathway, ErbB signaling pathway, Rap1 signaling pathway, Ras signaling pathway, NF signaling pathway, FoxO signaling pathway, VEGF signaling pathway, Apoptosis, MAPK signaling pathway, HIF-1 signaling pathway, Toll-like receptor signaling pathway, T cell receptor signaling pathway (Fig. 5b).

\section{Danhong injection attenuates bleomycin-induced PF in mice}

The mouse model of bleomycin-induced PF has a large amount of inflammatory factors released, especially in the pre-fibrotic stage of lung formation[9]. After modelling, we selected the same tissues from the same areas for HE and Masson staining, and found that the alveolar structure of the mice was obviously destroyed, with a large number of inflammatory cells infiltrating and a large amount of blue collagen deposited in the alveolar septum and interstitial lung. Danhong injection was able to alleviate the inflammation and collagen deposition, and the effect was more obvious at higher doses (Fig. 6a). Danhong injection had no effect on the body weight of mice with PF (Fig. 6b). Testing of lung function in mice revealed that Danhong injection was able to improve lung function and increase lung compliance by reducing lung resistance (Fig. $6 c, d)$.

\section{Dan Hong Injection reduced collagen deposition in the lungs of mice with}

\section{bleomycin-induced PF}

Collagen content in lung tissue is a key indicator for evaluating $P F[10]$. Immunohistochemical results showed that collagen deposition was significant in the 
model group compared to the normal control group and was improved in the high-dose group of mice after intervention with Danhong injection, with no significant difference in the low-dose group (Fig. 7a, b, c). Hydroxyproline is a non-essential amino acid found in collagen and some other extracellular animal proteins and plays a crucial role in the synthesis of collagen and in the thermodynamic stability of the triple helix conformation of collagen and associated tissues[11]. The detection of hydroxyproline content in mouse lung tissues revealed that the highest level of hydroxyproline was found in the lung tissues of mice in the model group, and that the level of hydroxyproline in the lung tissues of mice decreased after intervention with high doses of Danhong injection (Fig. 7d). The above results suggest that Danhong injection is sufficient to reduce collagen deposition in mice with PF to a certain extent.

\section{Effect of Danhong injection on the transcription of key target genes in lung tissue}

\section{of mice with PF}

The above results have confirmed that Danhong injection can alleviate the inflammatory response and reduce collagen deposition to alleviate $\mathrm{PF}$, but its mechanism is still unclear. Based on the results of the raw signal analysis, we predicted the mechanism of the anti-PF effect of Danhong injection. The expression of key target genes in the results was analyzed by extensive qRT-PCR assays. The results suggested that the genes with differences were MDM2, IL-2, CCL5, AKT1, MMP9, CASP3, MMP2 (Fig. 8). 


\section{Danhong injection exerts antifibrotic effects via Pi3k/Akt signaling pathway}

We have screened the key targets of Danhong injection against PF by q-PCR, and combined with the results of KEGG enriched signaling pathway, we found that Pi3k/Akt signaling pathway has the largest difference, so we think Danhong injection may exert its anti-fibrotic effect through Pi3k/Akt signaling pathway. The highest protein content of $\mathrm{p}-\mathrm{Pi} 3 \mathrm{k}$ and $\mathrm{p}$-Akt was found in the lung tissues of mice in the model group compared with the normal group by Western Blotting; the protein expression of p-Pi3k and p-Akt was significantly reduced after the intervention of Danhong injection compared with the model group ((Fig. 9a, b, c). The Pi3k/Akt signaling pathway, as one of the important signal transduction pathways in cells, plays a key role in inhibiting apoptosis and promoting proliferation by influencing the activation status of various downstream effector molecules, and is closely related to the development of many human diseases[12,13]. Apoptosis is a spontaneous and programmed cell death process that occurs under normal physiological or pathological conditions and is tightly regulated by the body[14]. TUNEL staining is one of the classic methods to detect apoptosis[15]. The staining results showed that the number of apoptotic cells in the lung tissues of mice in the model group was significantly increased. After the intervention of Danhong injection, the number of apoptotic cells was significantly reduced (Fig. 9d).

Caspase-3, Caspase- 8 and Caspase- 9 are the signature proteins of apoptosis[16]. The detection of apoptosis marker proteins in mouse lung tissues revealed that high doses of Danhong injection could reduce the levels of Caspase-3, Caspase- 8 and Caspase- 9 in lung tissues of mice with PF (Fig. 9e, f, g). These evidences suggest that Danhong 
injection may exert its anti-PF effect by inhibiting cell apoptosis through Pi3k/Akt signaling pathway.

\section{Discussion}

The onset and progression of PF is mediated by complex factors[17]. In addition to fibroblast-related processes, lung inflammation and oxidative stress play an integral role in promoting the formation of $\mathrm{PF}[18]$. Danhong injection is a compound Chinese herbal injection, the first patented Chinese patent medicine in China to rapidly resolve systemic organ blood supply deficiencies and ischemic infarct disorders, and is commonly used in the treatment of cardiovascular and cerebrovascular diseases[19,20]. Previous studies have confirmed the clinical use of Danhong injection in the treatment of idiopathic PF. It is able to alleviate patients' clinical symptoms and lung function, and no adverse effects have been identified[7].

Based on the clear clinical effectiveness of Danhong injection, we used network pharmacology to explore the material basis and potential mechanisms of action. A topological analysis of the drug's active ingredient and target interaction network revealed that luteolin, baicalin, quercetin, kaempferol and $\beta$-sitosterol are the active ingredients that may play an important role in Danhong Injection. Luteolin is a flavonoid with anti-inflammatory and antioxidant properties. It has been reported that luteolin can alleviate PF in both in vitro and in vivo experiments[21]. Quercetin is a biologically active flavonoid and has been shown to inhibit the development of PF by promoting the apoptosis of lung fibroblasts[22]. Baicalein, a flavonoid with anti- 
inflammatory and anti-cancer effects, can reduce the conversion of pulmonary fibroblasts to pulmonary myofibroblasts by inhibiting miR-21 levels, thereby inhibiting PF[23]. Kaempferol, a flavonoid widely found in plants and fruits, has antiinflammatory and anti-fibrotic effects and has been shown to reduce silica-induced PF in mice by modulating autophagy[24]. $\beta$-sitosterol can enhance mitochondrial function by promoting internal mitochondrial membrane fluidity, thereby slowing the progression of $\mathrm{PF}[25,26]$. Based on the above results, we concluded that the main active ingredients of Danhong injection had anti-inflammatory, antioxidant and anti-fibrotic effects.

GO and KEGG enrichment showed that Danhong injection may act through the targets of MDM2, IL-2, CCL5, AKT1, MMP9, CASP3 and MMP2. The enrichment pathway results showed that Danhong injection may act through Pi3k/Akt signaling pathway, Jak-STAT signaling pathway, GnRH signaling pathway, ErbB signaling pathway, Rap1 signaling pathway, Ras signaling pathway, NF signaling pathway, FoxO signaling pathway, Apoptosis, MAPK signaling pathway and many other signaling pathways. We found a closer relationship with Pi3k/Akt signaling pathway by extensive qRT-PCR and protein blotting methods. Danhong injection significantly reduced the protein expression of phosphorylated Pi3k and Akt in lung tissue of mice with PF.

The Pi3k protein family is involved in the regulation of various cellular functions such as cell proliferation, differentiation, apoptosis and glucose transport[27]. The Pi3k/Akt signaling pathway was found to activate the receptor tyrosine kinase, which translocated Pi3k from the cytoplasm to the cell membrane, activating and catalyzing 
the subunit that generates the 2nd messenger $\mathrm{PI}(3,4,5) \mathrm{P} 3$ that binds to the $\mathrm{PH}$ domain at the amino acid terminus of Akt and regulates the expression of the mammalian target of rapamycin downstream[28]. The high expression of NF-кB in early stages of PF is associated with activation of the Pi3k/Akt signaling pathway, and the interaction between the activated MAPK signaling pathway and Pi3k/Akt signaling pathway promotes PF formation. Inhibition of PF by blocking the Pi3k/Akt signaling pathway could potentially be a new direction for the treatment of PF. It was found that Pi3k inhibitors exhibited superior proliferation inhibitory activity against mouse MLG2908 lung fibroblasts and were able to improve lung function and pathological damage in mice with PF, and reduce hydroxyproline content and $\alpha$-SMA levels in lung tissue[29,30]. Our results have demonstrated that Danhong injection can attenuate the protein expression of phosphorylated Pi3k and Akt in the lung tissue of PF mice.

Activation of Akt by Pi3k can mediate cell growth induced by insulin, various growth factors, etc. through phosphorylation or inhibition of its downstream target proteins Bad, Caspase9, NF-кB, Forkhead, mTOR, Par-4 and P21, thus promoting cell survival via multiple pathways and exerting anti-apoptotic effects[31-34]. the TUNEL staining results demonstrated that Danhong injection The TUNEL staining results demonstrated that Danhong injection was able to reduce apoptosis in lung tissues. The reduction of Caspase-3, Caspase- 8 and Caspase- 9 proteins in lung tissues after administration also verified this conclusion. Our results confirm that Danhong injection can inhibit apoptosis through the Pi3k/Akt signaling pathway to exert anti-PF effects. However, fibroblasts and epithelial cells play an important role in pulmonary fibrosis, and lung 
tissue contains many types of cells, in which cell apoptosis occurs is currently unknown and this will be the direction of our future research.

\section{Conclusions}

In summary, this study was based on a network pharmacology approach to analyze the anti-PF mechanism of Danhong injection with multiple components-multiple targetsmultiple pathways-multiple effects, as well as experimental validation. The results suggest that Danhong injection may exert its anti-PF effect by regulating apoptosis through Pi3k/Akt signaling pathway, with a view to providing theoretical and experimental basis for the clinical application of Danhong injection.

\section{Abbreviations}

PF: Pulmonary fibrosis; TCMSP: Traditional Chinese Medicine Systems Pharmacology Database and Analysis Platform; TCMID: Traditional Chinese Medicines Integrated Database; SEA: Similarity ensemble approach; UniProt: Universal Protein; DAVID: DAVID Bioinformatics Resources; STRING: STRING databases; OMIM: Online Mendelian Inheritance in Man; CTD: Comparative Toxicogenomics Database; TTD: Therapeutic Target Database; H\&E: Haematoxylin and eosin; IHC: Immunohistochemistry; MF: Molecular Function; BP: Biological Process; CC: Cellular Components; qRT-PCR: RNA isolation and quantitative real-time PCR; WB: Western Blotting; ELISA: Enzyme-linked immunosorbent assay; TUNE: Terminal deoxynucleotidyl transferase dUTP nick end labelling; PVDF: Polyvinylidene difuoride; HRP: Horseradish peroxidase; RT: Room temperature ANOVA: A one-way analysis of variance. 


\section{Authors' contributions}

$\mathrm{XZ}$ and JS designed the study; DH and YX wrote the main manuscript text; DW, TH, DH, and YX performed the animal experiments; WP, YX, and DH analysed the data and prepared the figures. All authors read and approved the final manuscript.

\section{Funding}

This work was supported by National Natural Science Foundation of China (82074358), Jiangsu Provinces colleges and universities (integration of Chinese and Western medicine)

\section{Availability of data and materials}

The datasets used in the current study are available from the corresponding author on reasonable request.

\section{Ethics approval and consent to participate}

The animal care and experimental procedures used in the current study were approved by the Institutional Animal Care and Use Committee of the Nanjing University of Chinese Medicine.

\section{Consent for publication}

Not applicable.

\section{Competing interests}

The authors declare that they have no competing interests. 


\section{Author details}

${ }^{1}$ Affiliated Hospital of Nanjing University of Chinese Medicine, Nanjing, 210023 China.

${ }^{2}$ Department of Respiratory Medicine, Jiangsu Province Hospital of Chinese Medicine, Nanjing,

210023 China. ${ }^{3}$ Jiangsu Key Laboratory of Pediatric Respiratory Disease, Institute of Pediatrics,

Affiliated Hospital of Nanjing University of Chinese Medicine, No. 138, Xianlin Avenue, Qixia

District, Nanjing, 210023 China. ${ }^{4}$ Medical Metabolomics Center, Nanjing University of Chinese

Medicine, Nanjing, 210023 China

441

442

\section{References}

1. Spagnolo P, Kropski JA, Jones MG, Lee JS, Rossi G, Karampitsakos T, et al. Idiopathic pulmonary fibrosis: Disease mechanisms and drug development. Pharmacol Ther. 2021;222:107798.

2. Maher TM, Strek ME. Antifibrotic therapy for idiopathic pulmonary fibrosis: time to treat. Respir Res. 2019;20:205.

3. Sack C, Raghu G. Idiopathic pulmonary fibrosis: unmasking cryptogenic environmental factors. Eur Respir J. England; 2019;53.

4. Zhao Wenqing, Zheng Minyu. Traditional Chinese Medicine and Western Treatment Progress of IPF. Chinese Archives of Traditional Chinese Medicine. 2016;402-5.

5. Zhang Xinyue. Analysis of blood stasis throughout interstitial lung disease. Traditional Chinese Medicinal Research. 2013;1-2.

6. Insufficiency being the source of all diseases and Danhong Injection. Insufficiency being the source of all diseases and Danhong Injection. Clinical Journal of Chinese Medicine. 2015;65-7.

7. Xin Lili, Jiang Miao, Zhang Geng, Gong Jie-ning. Efficacy and safety of Danhong injection for idiopathic pulmonary fibrosis: Meta-analysis. China Journal of Chinese Materia Medica. 2016;3859-65.

8. Xu X, Zhang W, Huang C, Li Y, Yu H, Wang Y, et al. A novel chemometric method for the prediction of human oral bioavailability. Int J Mol Sci. 2012;13:6964-82.

9. Ng B, Dong J, D’Agostino G, Viswanathan S, Widjaja AA, Lim W-W, et al. 
Interleukin-11 is a therapeutic target in idiopathic pulmonary fibrosis. Sci Transl Med. United States; 2019;11.

10. Plantier L, Cazes A, Dinh-Xuan A-T, Bancal C, Marchand-Adam S, Crestani B. Physiology of the lung in idiopathic pulmonary fibrosis. Eur Respir Rev. England; 2018;27.

11. Srivastava AK, Khare P, Nagar HK, Raghuwanshi N, Srivastava R. Hydroxyproline: A Potential Biochemical Marker and Its Role in the Pathogenesis of Different Diseases. Curr Protein Pept Sci. United Arab Emirates; 2016;17:596-602.

12. Jafari M, Ghadami E, Dadkhah T, Akhavan-Niaki H. PI3k/AKT signaling pathway: Erythropoiesis and beyond. J Cell Physiol. United States; 2019;234:2373-85.

13. Rahmani M, Nkwocha J, Hawkins E, Pei X, Parker RE, Kmieciak M, et al. Cotargeting BCL-2 and PI3K Induces BAX-Dependent Mitochondrial Apoptosis in AML Cells. Cancer Res. 2018;78:3075-86.

14. Xu X, Lai Y, Hua Z-C. Apoptosis and apoptotic body: disease message and therapeutic target potentials. Biosci Rep. 2019;39.

15. Kyrylkova K, Kyryachenko S, Leid M, Kioussi C. Detection of apoptosis by TUNEL assay. Methods Mol Biol. United States; 2012;887:41-7.

16. Holmes WF, Soprano DR, Soprano KJ. Synthetic retinoids as inducers of apoptosis in ovarian carcinoma cell lines. J Cell Physiol. United States; 2004;199:317-29.

17. Spagnolo P, Kropski JA, Jones MG, Lee JS, Rossi G, Karampitsakos T, et al. Idiopathic pulmonary fibrosis: Disease mechanisms and drug development. Pharmacol Ther. 2021;222:107798.

18. Miao K, Pan T, Mou Y, Zhang L, Xiong W, Xu Y, et al. Scutellarein inhibits BLMmediated pulmonary fibrosis by affecting fibroblast differentiation, proliferation, and apoptosis. Ther Adv Chronic Dis. 2020;11:2040622320940185.

19. Wan L-M, Tan L, Wang Z-R, Liu S-X, Wang Y-L, Liang S-Y, et al. Preventive and therapeutic effects of Danhong injection on lipopolysaccharide induced acute lung injury in mice. J Ethnopharmacol. Ireland; 2013;149:352-9.

20. Feng X, Li Y, Wang Y, Li L, Little PJ, Xu S-W, et al. Danhong injection in cardiovascular and cerebrovascular diseases: Pharmacological actions, molecular mechanisms, and therapeutic potential. Pharmacol Res. Netherlands; 2019;139:62-75.

21. Chen C-Y, Peng W-H, Wu L-C, Wu C-C, Hsu S-L. Luteolin ameliorates experimental lung fibrosis both in vivo and in vitro: implications for therapy of lung fibrosis. J Agric Food Chem. United States; 2010;58:11653-61. 
22. Veith C, Drent M, Bast A, van Schooten FJ, Boots AW. The disturbed redox-balance in pulmonary fibrosis is modulated by the plant flavonoid quercetin. Toxicol Appl Pharmacol. United States; 2017;336:40-8.

23. Cui X, Sun X, Lu F, Jiang X. Baicalein represses TGF- $\beta 1$-induced fibroblast differentiation through the inhibition of miR-21. Toxicol Appl Pharmacol. United States; 2018;358:35-42.

24. Liu H, Yu H, Cao Z, Gu J, Pei L, Jia M, et al. Kaempferol Modulates Autophagy and Alleviates Silica-Induced Pulmonary Fibrosis. DNA Cell Biol. United States; 2019;38:1418-26.

25. Lee I-A, Kim E-J, Kim D-H. Inhibitory effect of $\beta$-sitosterol on TNBS-induced colitis in mice. Planta Med. Germany; 2012;78:896-8.

26. Shi $\mathrm{C}, \mathrm{Wu} \mathrm{F}, \mathrm{Xu}$ J. Incorporation of $\beta$-sitosterol into mitochondrial membrane enhances mitochondrial function by promoting inner mitochondrial membrane fluidity. J Bioenerg Biomembr. United States; 2013;45:301-5.

27. Su D, Zhou Y, Hu S, Guan L, Shi C, Wang Q, et al. Role of GAB1/PI3K/AKT signaling high glucose-induced cardiomyocyte apoptosis. Biomed Pharmacother. France; 2017;93:1197-204.

28. Mercer PF, Woodcock HV, Eley JD, Platé M, Sulikowski MG, Durrenberger PF, et al. Exploration of a potent PI3 kinase/mTOR inhibitor as a novel anti-fibrotic agent in IPF. Thorax. 2016;71:701-11.

29. Lin S, Jin J, Liu Y, Tian H, Zhang Y, Fu R, et al. Discovery of 4-Methylquinazoline Based PI3K Inhibitors for the Potential Treatment of Idiopathic Pulmonary Fibrosis. J Med Chem. United States; 2019;62:8873-9.

30. Hettiarachchi SU, Li Y-H, Roy J, Zhang F, Puchulu-Campanella E, Lindeman SD, et al. Targeted inhibition of PI3 kinase/mTOR specifically in fibrotic lung fibroblasts suppresses pulmonary fibrosis in experimental models. Sci Transl Med. United States; $2020 ; 12$.

31. Jia G, Mitra AK, Gangahar DM, Agrawal DK. Insulin-like growth factor-1 induces phosphorylation of PI3K-Akt/PKB to potentiate proliferation of smooth muscle cells in human saphenous vein. Exp Mol Pathol. 2010;89:20-6.

32. Jung F, Haendeler J, Goebel C, Zeiher AM, Dimmeler S. Growth factor-induced phosphoinositide 3-OH kinase/Akt phosphorylation in smooth muscle cells: induction of cell proliferation and inhibition of cell death. Cardiovasc Res. England; 2000;48:148-57.

33. Xing Y-Q, Li A, Yang Y, Li X-X, Zhang L-N, Guo H-C. The regulation of FOXO1 and its role in disease progression. Life Sci. Netherlands; 2018;193:124-31. 
34. Zhang L-N, Zhao L, Yan X-L, Huang Y-H. Loss of G3BP1 suppresses proliferation, migration, and invasion of esophageal cancer cells via Wnt/ $\beta$-catenin and PI3K/AKT signaling pathways. J Cell Physiol. United States; 2019;234:20469-84.

\section{Figures}

\section{Figure 1}

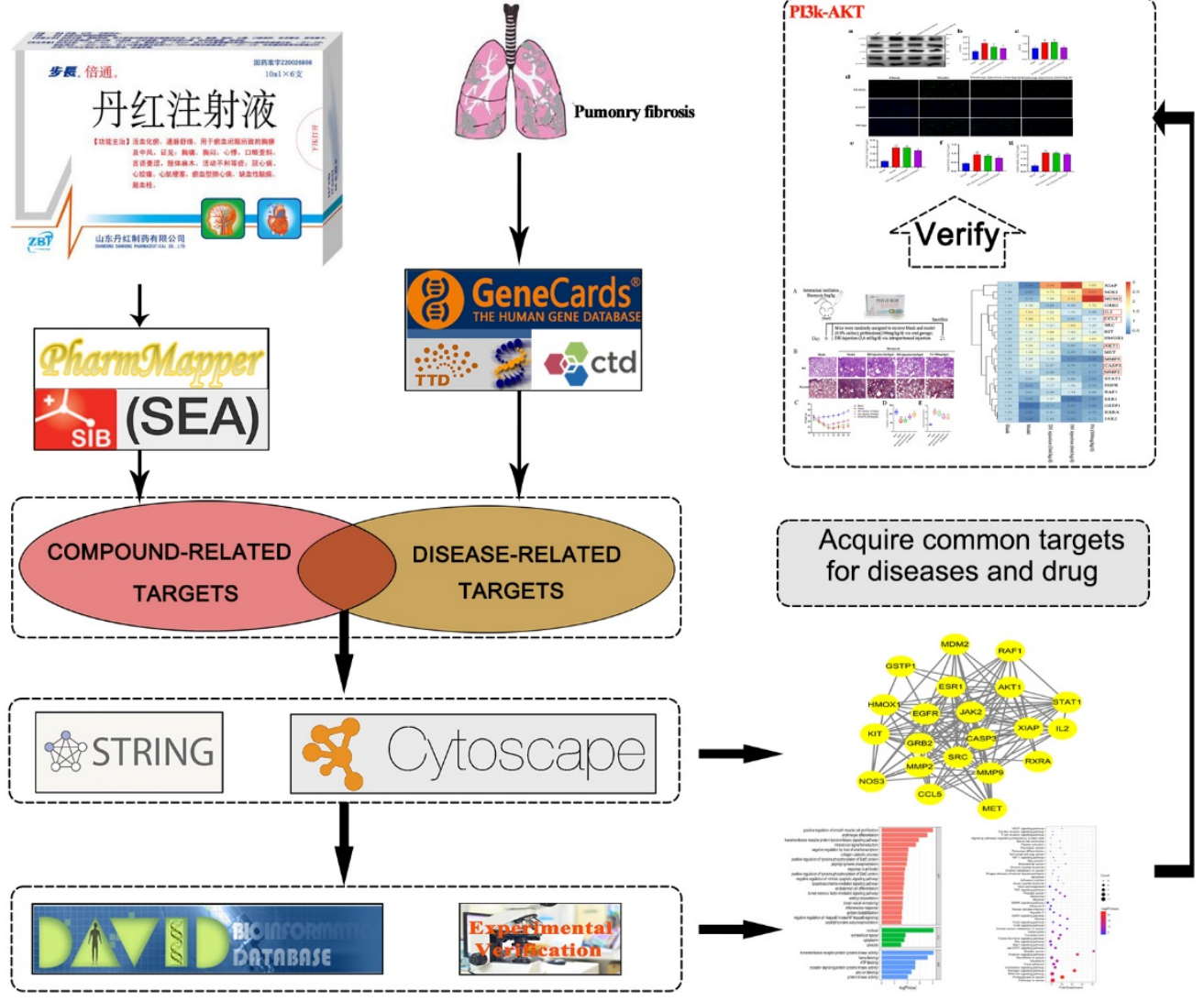

Fig. 1 Technological road-map 
Figure 2

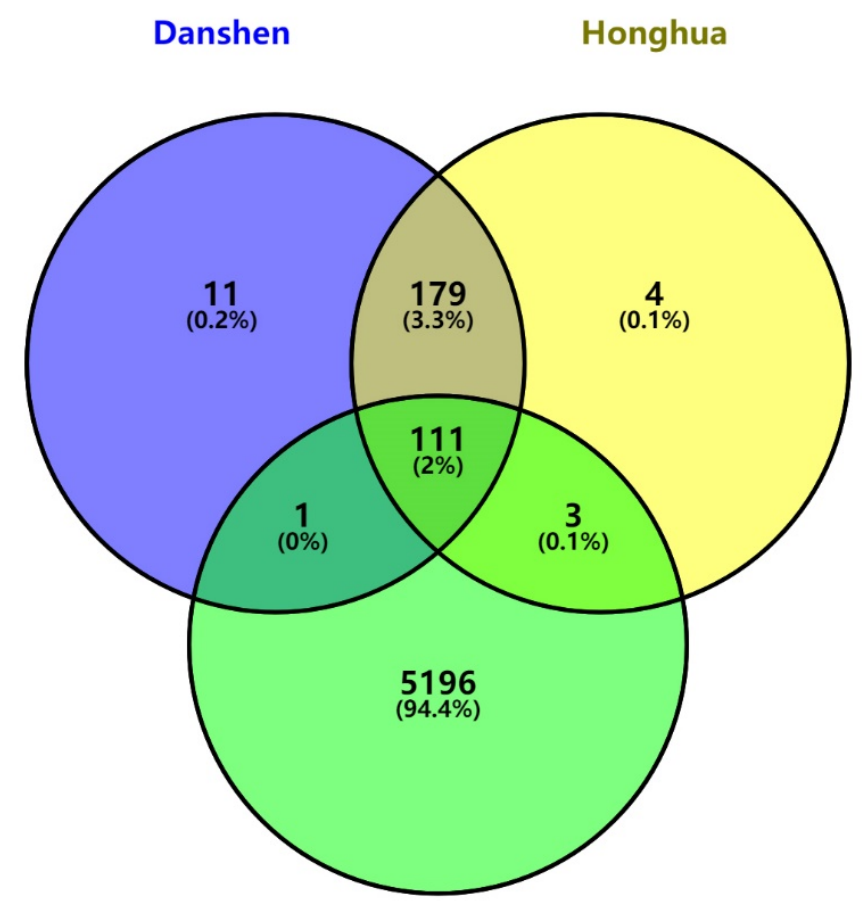

Pulmomary fibrosis

557

558

Fig. 2 Drug-disease intersection targets

559

560

561

562

563

564

565

566

567

568

569 
570

571

572

573

574

575

576

577

578

579

580

581

582

583

584

585

586

587

588

589

590

591

592

593

594

\section{Figure 3}

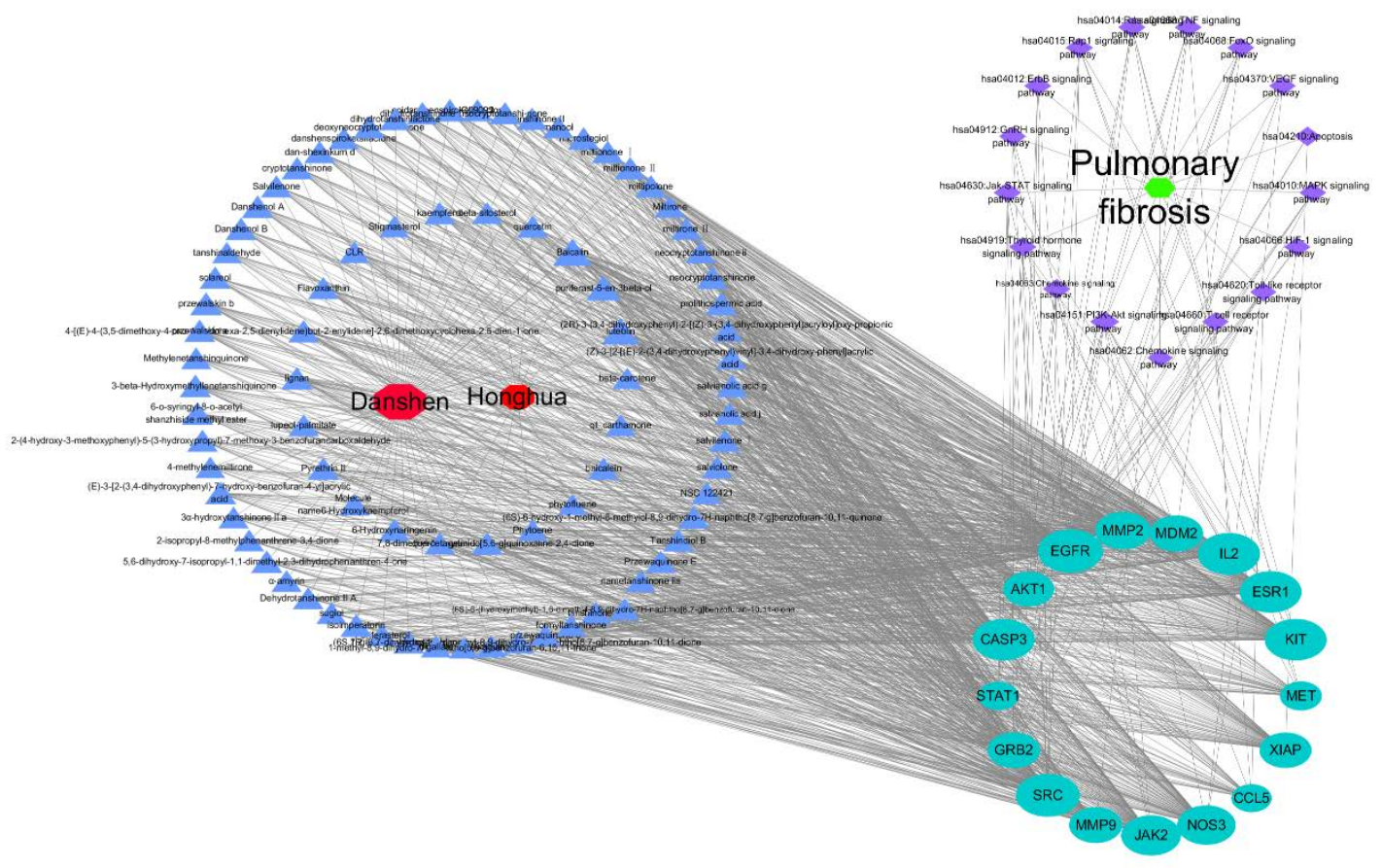

Fig 3 Anti-PF network diagram of the active ingredients of Danhong injection 
Figure 4

596

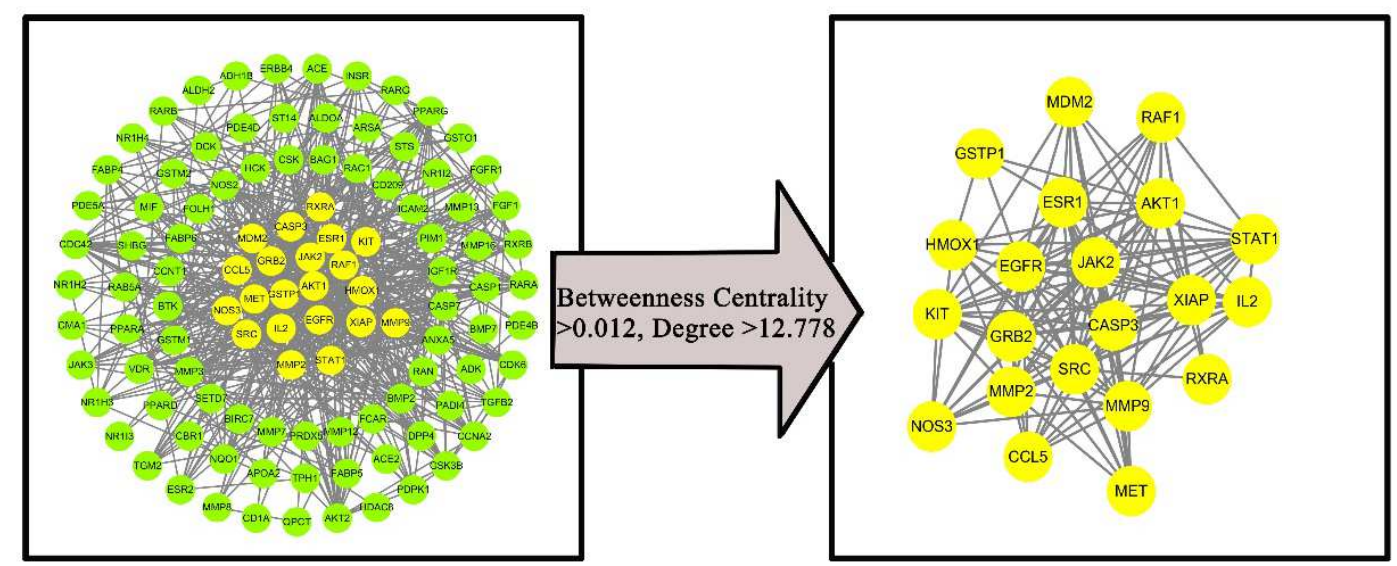

597

Fig. 4 Screening of the core targets of the active ingredient of Danhong injection against PF

598

599

600

601

602

603

604

605

606

607

608

609

610

611

612

613

614

615

616

617

618

619

620

621

622

623

624

625

626

627 
628

Figure 5

a

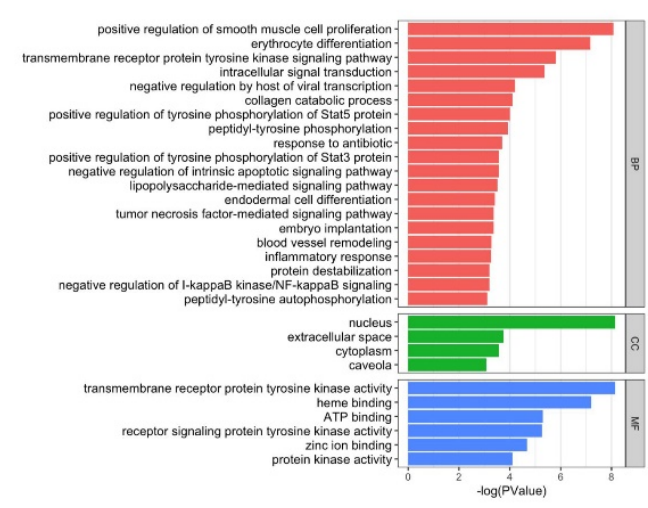

629

630

631

632

633

634

635

636

637

638

639

640

641

642

643

644

645

646

647

648

649

650

651

652

653

654

655

656 analysis; b KEGG enrichment analysis b

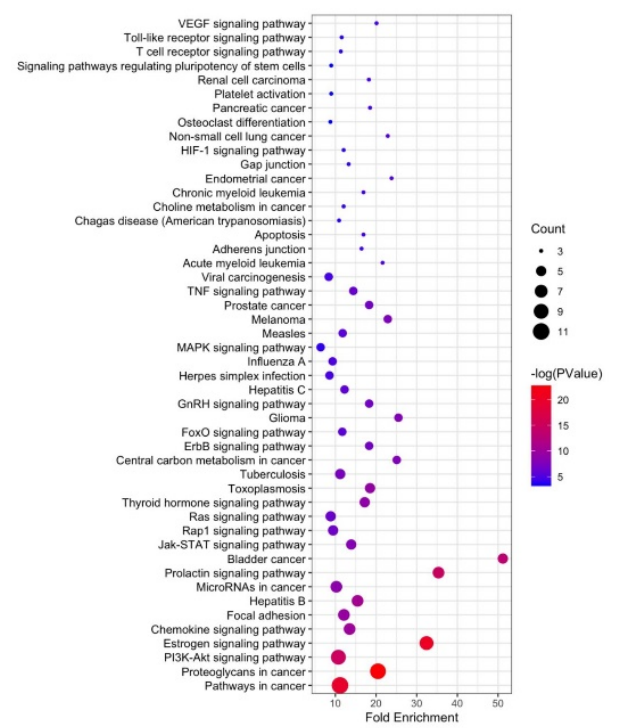

Fig. 5 Anti-PF enrichment analysis of the active ingredients of Danhong injection. a GO enrichment 
Figure 6

$\mathbf{a}$

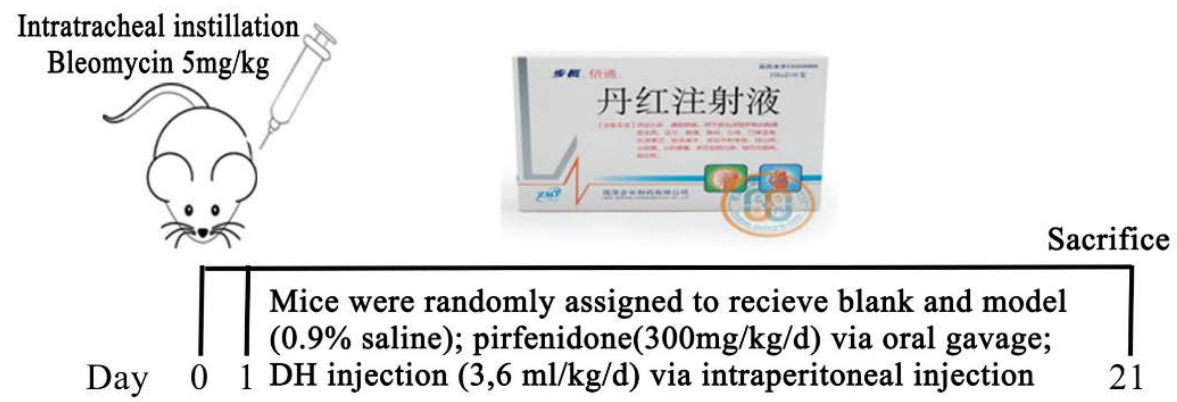

$\mathbf{b}$

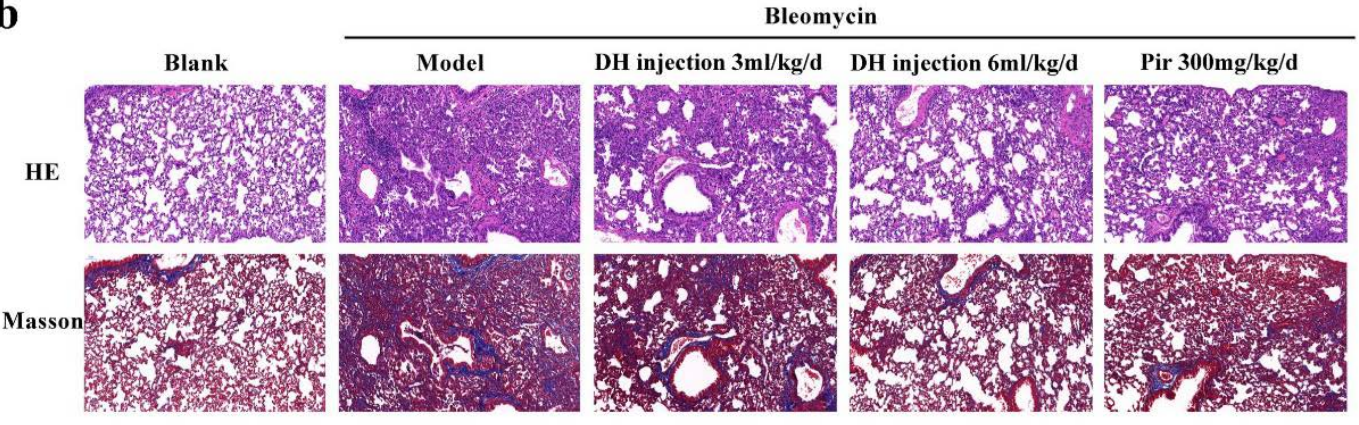

c

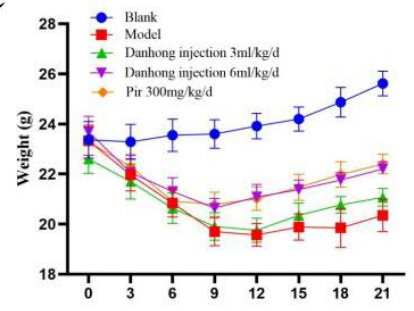

658

659 d

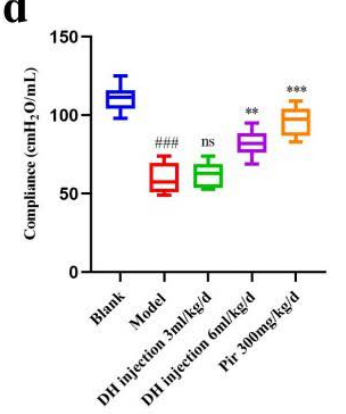

e

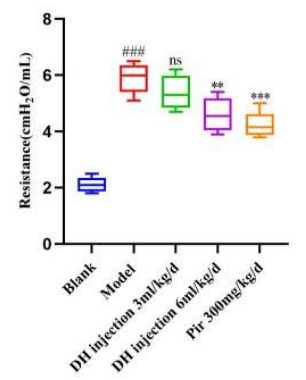

Fig. 6 Danhong injection attenuates bleomycin-induced PF in mice. a Modeling diagram. b. Image of H\&E staining and Masson staining obtained at 20xmagnifcation in the lung. c Body weight. $d$ Lung compliance. e Lung resistance. All of the data are expressed as mean $\pm \mathrm{SD}(\mathrm{n}=6),{ }^{\# \#+} \mathrm{P}<0.01$ compared with blank group ${ }^{*} \mathrm{P}<0.05$ compared with model group, ${ }^{* *} \mathrm{P}<0.01$ compared with model group, ${ }^{* * *} \mathrm{P}<0.001$ compared with model group and $\mathrm{ns}=$ no significant compared with model group. Scale bar $=50 \mu \mathrm{m}$ 
Figure 7

a

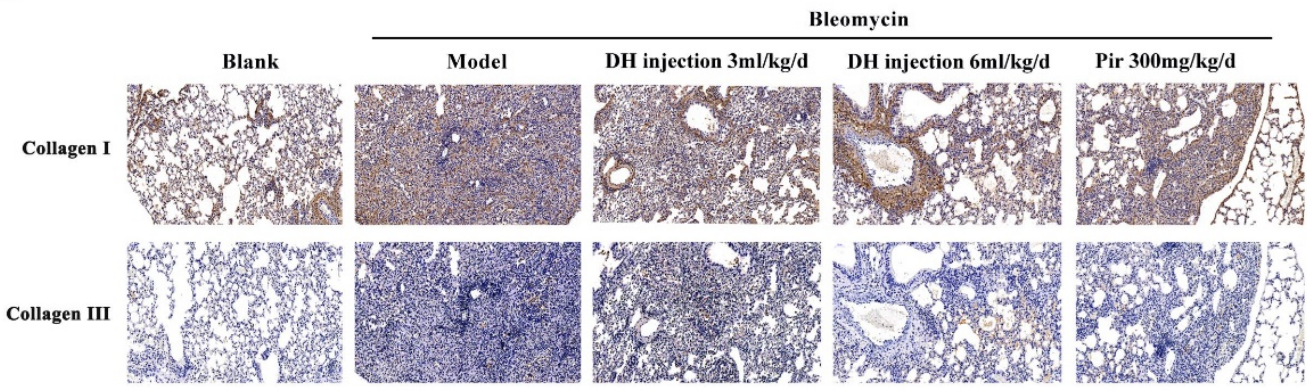

b

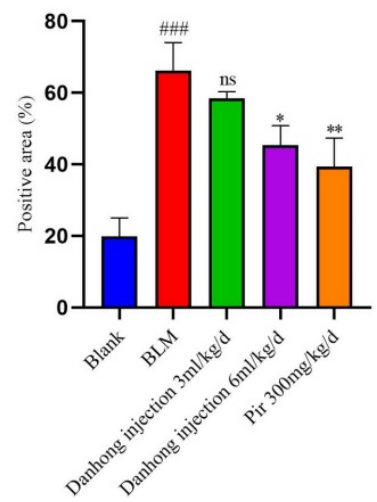

c

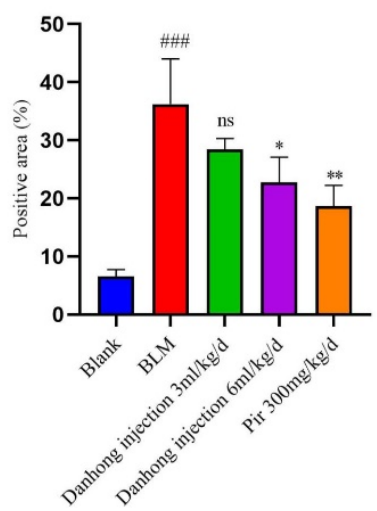

d

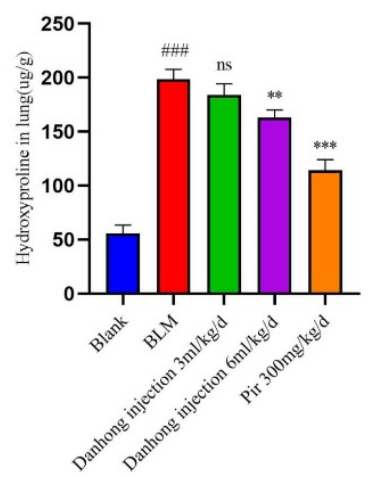

Fig.7 Dan Hong Injection reduced collagen deposition in the lungs of mice with bleomycin-induced PF. a. immunohistochemistry staining of Collagen I and collagen III in lung and image of immunohistochemistry obtained at 20xmagnifcation in lung. b, c Histology score of the pulmonary fibrosis calculated by Collagen I and collagen III. d Contents of Hydroxyproline in lung tissue. All of the data are expressed as mean $\pm \mathrm{SD}(\mathrm{n}=6),{ }^{\# \#} \mathrm{P}<0.001$ compared with blank group ${ }^{*} \mathrm{P}<0.5$ compared with model group, ${ }^{* *} \mathrm{P}<0.01$ compared with model group, ${ }^{* * *} \mathrm{P}<0.001$ compared with model group and $\mathrm{ns}=$ no significant compared with model group. Scale bar=50 $\mu \mathrm{m}$ 
Figure 8

Fig.8 Effect of Danhong injection on the transcription of key target genes in lung tissue of mice with PF. Real-time qPCR analysis of genes, relative expression of XIAP, NOS3, MDM2, GRB2, IL-2, CCL5, SRC, KIT, HMOX1, AKT1, MET, MMP9, CASP3, MMP2, STAT1, EGFR, RAF1, ESR1, GSTP1, RXRA, JAK2 and make a heatmap. All of the data are expressed as mean \pm SD $(\mathrm{n}=3)$. 
Figure 9
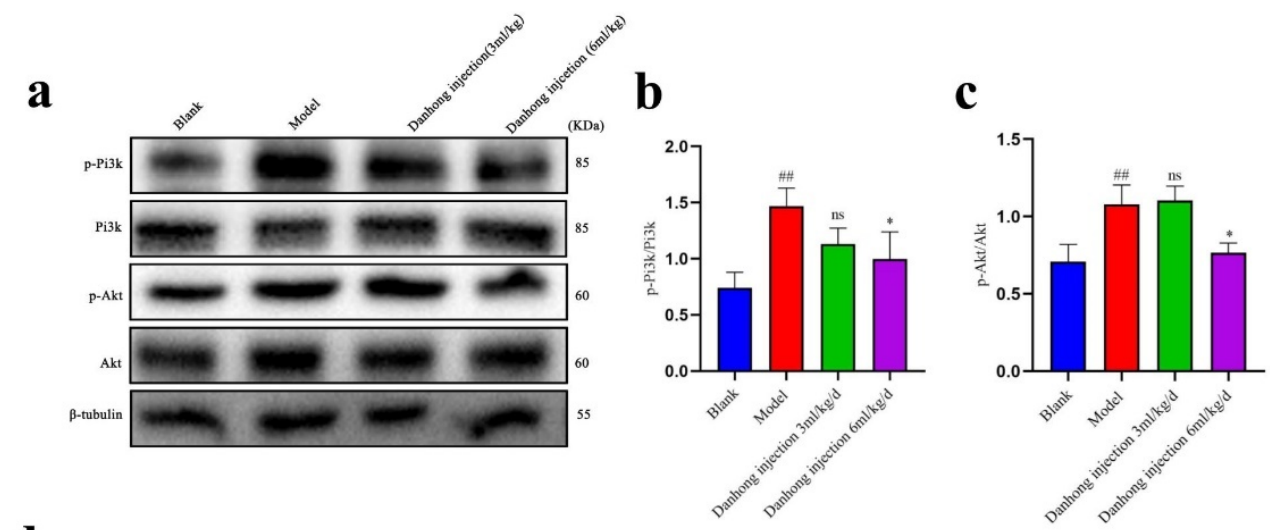

d

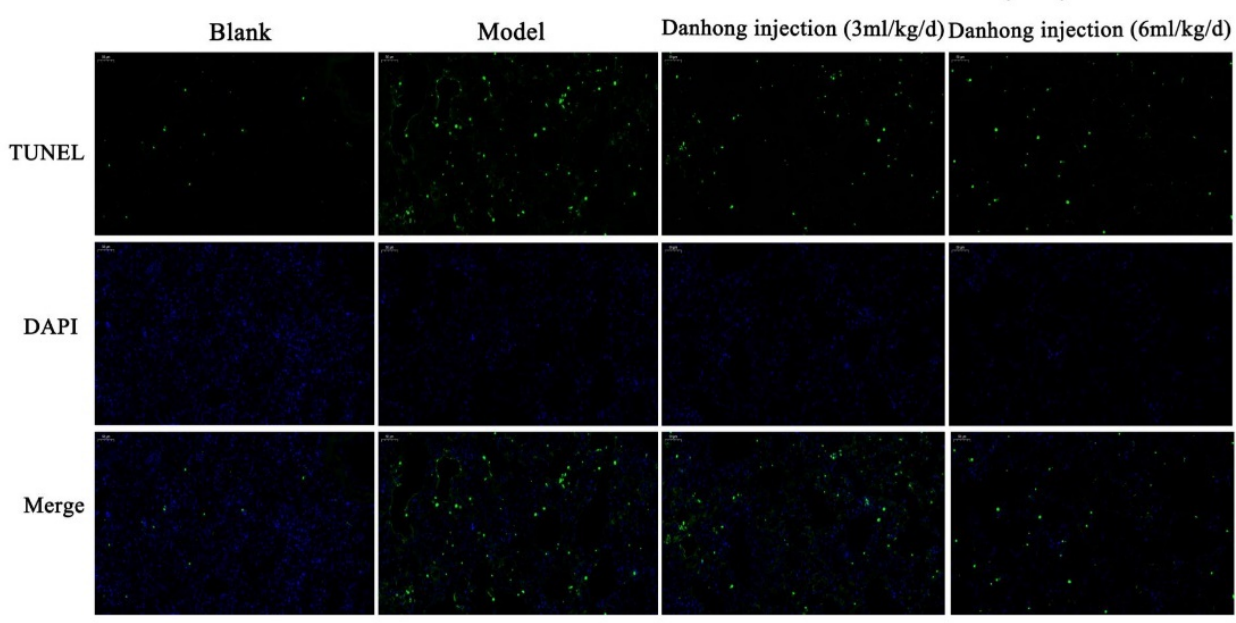

$\mathbf{e}$

f
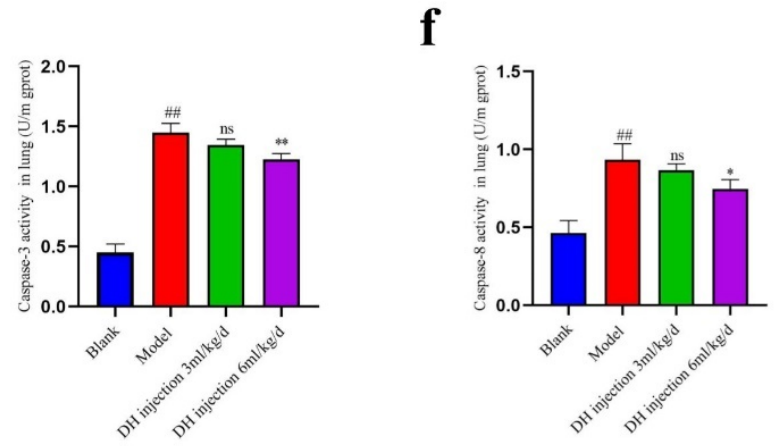

$\mathbf{g}$

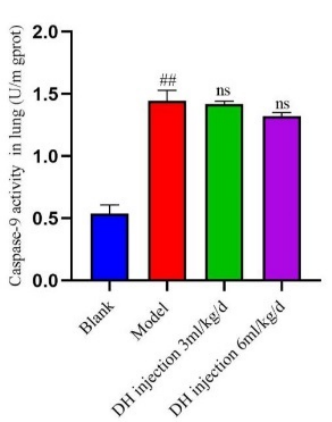

Fig.9 Danhong injection exerts antifibrotic effects via Pi3k/Akt signaling pathway. a b, c Western blotting of p-Pi3k, Pi3k, p-Akt, Akt, and $\beta$-tubulin protein in lung. $\mathrm{d}$ Immunofluorescence staining of apoptotic cell. DAPI was used to stain the nucleus. d, e, f Contents of Caspase -3 , Caspase-8、Caspase-9 in lung tissue. All of the data are expressed as mean $\pm \mathrm{SD}(\mathrm{n}=3),{ }^{\#} \mathrm{P}<0.01$ compared with blank group ${ }^{*} \mathrm{P}<0.05$ compared with model group ${ }^{* *} \mathrm{P}<0.01$ compared with model group and ns $=$ no significant compared with model group. Scale bar $=50 \mu \mathrm{m}$ 
Tables

Table 1 Primer sequences. This is where the description of the table should go

\begin{tabular}{lll}
\hline Gene & Forward Primer & Reveres Primer \\
\hline GAPDH & GGTTGTCTCCTGCGACTTCA & TGGTCCAGGGTTTCTTACTCC \\
XIAP & ATATGAAGCACGGATCGTTACT & CTTATCGCCTTCACCTAAAGC \\
NOS3 & CTGAGAGCCTGCAATTACTACC & TTTCCACAGAGAGGATTGTAGC \\
MDM2 & TCTGATCAGTTTAGCGTGGAAT & ATCGCTTTCTCCTGTCTGATAG \\
GRB2 & ATAAGGCAGAACTCAATGGGAA & ACATCATTTCCAAACTTGACGG \\
IL-2 & TGAGCAGGATGGAGAATTACAG & CAGAGGTCCAAGTTCATCTTCT \\
CCL5 & GTATTTCTACACCAGCAGCAAG & TCTTGAACCCACTTCTTCTCTG \\
SRC & CTATGTGGAGCGGATGAACTAT & ATTCGTTGTCTTCTATGAGCCG \\
KIT & CAAGAGTTCCGCCTTCTTTAAC & GTGATCATAAGGAAGTTGCGTC \\
HMOX1 & TCCTTGTACCATATCTACACGG & GAGACGCTTTACATAGTGCTGT \\
AKT1 & TGCACAAACGAGGGGAATATAT & CGTTCCTTGTAGCCAATAAAGG \\
MET & TCACTATCTACCTGTTGCAAGG & CAGCATTTTAGCATCACTTCGT \\
MMP9 & CAAAGACCTGAAAACCTCCAAC & GACTGCTTCTCTCCCATCATC \\
CASP3 & GAAACTCTTCATCATTCAGGCC & GCGAGTGAGAATGTGCATAAAT \\
MMP2 & ACTTTGAGAAGGATGGCAAGTA & CTTCTTATCCCGGTCATAGTCC \\
STAT1 & GATCCGTCAGCAGCTTAAAAAG & CTGACAACACCTGCTTGTTTTT \\
EGFR & TGAGTTCTCTGAGTGCAACTAG & GAATGCGTCATCTATGTTGTCC \\
RAF1 & ACATCAACAACCGAGACCAGATCATC & CACAGTCAGCCACCAACCTCTTC \\
ESR1 & CTACTACCTGGAGAACGAGC & GCGTCGATTGTCAGAATTAGAC \\
GSTP1 & AGCTGGAAGGAGGAGGTGGTTAC & AGGTGTCTCAAGATGGCATTAGATTGG \\
RXRA & GAGCAGCACTGAGGATATCAAG & GAAGGAGGCCATATTTCCTGAG \\
JAK2 & GAGCAGCACTGAGGATATCAAG & GAAGGAGGCCATATTTCCTGAG \\
\hline
\end{tabular}

\section{3}

734 Table 2 Active ingredients of compounds. This is where the description of the table 735 should go

\begin{tabular}{|c|c|c|c|c|}
\hline Molecular ID & Chemical name & OB\% & DL & Herbal \\
\hline MOL001601 & 1,2,5,6-tetrahydrotanshinone & 38.75 & 0.36 & Radix Salviae \\
\hline MOL001659 & Poriferasterol & 43.83 & 0.76 & Radix Salviae \\
\hline MOL001771 & poriferast-5-en-3beta-ol & 36.91 & 0.75 & Radix Salviae \\
\hline MOL001942 & isoimperatorin & 45.46 & 0.23 & Radix Salviae \\
\hline MOL002222 & sugiol & 36.11 & 0.28 & Radix Salviae \\
\hline MOL002651 & Dehydrotanshinone II A & 43.76 & 0.4 & Radix Salviae \\
\hline \multirow[t]{2}{*}{ MOL002776 } & Baicalin & 40.12 & 0.75 & Radix Salviae \\
\hline & & & & Carthami Flos \\
\hline MOL000569 & digallate & 61.85 & 0.26 & Radix Salviae \\
\hline \multirow[t]{2}{*}{ MOL000006 } & luteolin & 36.16 & 0.25 & Radix Salviae \\
\hline & & & & Carthami Flos \\
\hline MOL006824 & $\alpha$-amyrin & 39.51 & 0.76 & Radix Salviae \\
\hline MOL007036 & 5,6-dihydroxy-7-isopropyl-1,1-dimethyl-2,3-dihydrophenanthren-4-one & 33.77 & 0.29 & Radix Salviae \\
\hline MOL007041 & 2-isopropyl-8-methylphenanthrene-3,4-dione & 40.86 & 0.23 & Radix Salviae \\
\hline
\end{tabular}




\begin{tabular}{|c|c|c|c|c|}
\hline MOL007045 & $3 \alpha$-hydroxytanshinone II a & 44.93 & 0.44 & Radix Salviae \\
\hline MOL007048 & (E)-3-[2-(3,4-dihydroxyphenyl)-7-hydroxy-benzofuran-4-yl]acrylic acid & 48.24 & 0.31 & Radix Salviae \\
\hline MOL007049 & 4-methylenemiltirone & 34.35 & 0.23 & Radix Salviae \\
\hline MOL007050 & 2-(4-hydroxy-3-methoxyphenyl)-5-(3-hydroxypropyl)-7-methoxy-3- & 62.78 & 0.4 & Radix Salviae \\
\hline \multicolumn{5}{|c|}{ benzofurancarboxaldehyde } \\
\hline MOL007051 & 6-o-syringyl-8-o-acetyl shanzhiside methyl ester & 46.69 & 0.71 & Radix Salviae \\
\hline MOL007058 & formyltanshinone & 73.44 & 0.42 & Radix Salviae \\
\hline MOL007059 & 3-beta-Hydroxymethyllenetanshiquinone & 32.16 & 0.41 & Radix Salviae \\
\hline MOL007061 & Methylenetanshinquinone & 37.07 & 0.36 & Radix Salviae \\
\hline MOL007063 & przewalskin a & 37.11 & 0.65 & Radix Salviae \\
\hline MOL007064 & przewalskin $b$ & 110.32 & 0.44 & Radix Salviae \\
\hline MOL007068 & Przewaquinone B & 62.24 & 0.41 & Radix Salviae \\
\hline MOL007069 & przewaquinone $\mathrm{c}$ & 55.74 & 0.4 & Radix Salviae \\
\hline MOL007070 & (6S,7R)-6,7-dihydroxy-1,6-dimethyl-8,9-dihydro-7H-naphtho[8,7-g]benzofuran-10,11- & 41.31 & 0.45 & Radix Salviae \\
\hline \multicolumn{5}{|c|}{ dione } \\
\hline MOL007071 & przewaquinone $\mathrm{f}$ & 40.31 & 0.46 & Radix Salvia \\
\hline MOL007077 & sclareol & 43.67 & 0.21 & Radix Salvia \\
\hline MOL007079 & tanshinaldehyde & 52.47 & 0.45 & Radix Salvia \\
\hline MOL007081 & Danshenol B & 57.95 & 0.56 & Radix Salvia \\
\hline MOL007082 & Danshenol A & 56.97 & 0.52 & Radix Salvia \\
\hline MOL007085 & Salvilenone & 30.38 & 0.38 & Radix Salvia \\
\hline MOL007088 & cryptotanshinone & 52.34 & 0.4 & Radix Salvia \\
\hline MOL007093 & dan-shexinkum d & 38.88 & 0.55 & Radix Salvia \\
\hline MOL007094 & danshenspiroketallactone & 50.43 & 0.31 & Radix Salvia \\
\hline MOL007098 & deoxyneocryptotanshinone & 49.4 & 0.29 & Radix Salvia \\
\hline MOL007100 & dihydrotanshinlactone & 38.68 & 0.32 & Radix Salvia \\
\hline MOL007101 & dihydrotanshinone I 278.32 & 45.04 & 0.36 & Radix Salvia \\
\hline MOL007105 & epidanshenspiroketallactone & 68.27 & 0.31 & Radix Salviac \\
\hline MOL007107 & C09092 & 36.07 & 0.25 & Radix Salvia \\
\hline MOL007108 & isocryptotanshi-none & 54.98 & 0.39 & Radix Salvia \\
\hline MOL007111 & Isotanshinone II & 49.92 & 0.4 & Radix Salvia \\
\hline MOL007115 & manool & 45.04 & 0.2 & Radix Salvia \\
\hline MOL007118 & microstegiol & 39.61 & 0.28 & Radix Salvia \\
\hline MOL007119 & miltionone I & 49.68 & 0.32 & Radix Salvia \\
\hline MOL007120 & miltionone II & 71.03 & 0.44 & Radix Salvia \\
\hline MOL007121 & miltipolone & 36.56 & 0.37 & Radix Salvia \\
\hline MOL007122 & Miltirone & 38.76 & 0.25 & Radix Salvia \\
\hline MOL007123 & miltirone II & 44.95 & 0.24 & Radix Salvia \\
\hline MOL007124 & neocryptotanshinone ii & 39.46 & 0.23 & Radix Salvia \\
\hline MOL007125 & neocryptotanshinone & 52.49 & 0.32 & Radix Salvia \\
\hline MOL007127 & 1-methyl-8,9-dihydro-7H-naphtho[5,6-g]benzofuran-6,10,11-trione & 34.72 & 0.37 & Radix Salvia \\
\hline MOL007130 & prolithospermic acid & 64.37 & 0.31 & Radix Salvia \\
\hline MOL007132 & (2R)-3-(3,4-dihydroxyphenyl)-2-[(Z)-3-(3,4-dihydroxyphenyl)acryloyl]oxy-propionic & 109.38 & 0.35 & Radix Salvia \\
\hline
\end{tabular}


Page 34 of 34

\begin{tabular}{|c|c|c|c|c|}
\hline MOL007140 & (Z)-3-[2-[(E)-2-(3,4-dihydroxyphenyl)vinyl]-3,4-dihydroxy-phenyl]acrylic acid & 88.54 & 0.26 & Radix Salviae \\
\hline MOL007141 & salvianolic acid $g$ & 45.56 & 0.61 & Radix Salviae \\
\hline MOL007142 & salvianolic acid $\mathrm{j}$ & 43.38 & 0.72 & Radix Salviae \\
\hline MOL007143 & salvilenone I & 32.43 & 0.23 & Radix Salviae \\
\hline MOL007145 & salviolone & 31.72 & 0.24 & Radix Salviae \\
\hline MOL007149 & NSC 122421 & 34.49 & 0.28 & Radix Salviae \\
\hline MOL007150 & $\begin{array}{c}\text { (6S)-6-hydroxy-1-methyl-6-methylol-8,9-dihydro-7H-naphtho[8,7-g]benzofuran-10,11- } \\
\text { quinone }\end{array}$ & 75.39 & 0.46 & Radix Salviae \\
\hline MOL007151 & Tanshindiol B & 42.67 & 0.45 & Radix Salviae \\
\hline MOL007152 & Przewaquinone E & 42.85 & 0.45 & Radix Salviae \\
\hline MOL007154 & tanshinone iia & 49.89 & 0.4 & Radix Salviae \\
\hline MOL007155 & $\begin{array}{c}\text { (6S)-6-(hydroxymethyl)-1,6-dimethyl-8,9-dihydro-7H-naphtho[8,7-g]benzofuran-10,11- } \\
\text { dione }\end{array}$ & 65.26 & 0.45 & Radix Salviae \\
\hline MOL007156 & tanshinone VI & 45.64 & 0.3 & Radix Salviae \\
\hline MOL001771 & poriferast-5-en-3beta-ol & 36.91 & 0.75 & Carthami Flos \\
\hline MOL002680 & Flavoxanthin & 60.41 & 0.56 & Carthami Flos \\
\hline MOL002694 & $\begin{array}{l}\text { 4-[(E)-4-(3,5-dimethoxy-4-oxo-1-cyclohexa-2,5-dienylidene)but-2-enylidene]-2,6- } \\
\text { dimethoxycyclohexa-2,5-dien-1-one }\end{array}$ & 48.47 & 0.36 & Carthami Flos \\
\hline MOL002695 & lignan & 43.32 & 0.65 & Carthami Flos \\
\hline MOL002698 & lupeol-palmitate & 33.98 & 0.32 & Carthami Flos \\
\hline MOL002706 & Phytoene & 39.56 & 0.5 & Carthami Flos \\
\hline MOL002707 & phytofluene & 43.18 & 0.5 & Carthami Flos \\
\hline MOL002710 & Pyrethrin II & 48.36 & 0.35 & Carthami Flos \\
\hline MOL002712 & 6-Hydroxykaempferol & 62.13 & 0.27 & Carthami Flos \\
\hline MOL002714 & baicalein & 33.52 & 0.21 & Carthami Flos \\
\hline MOL002717 & qt_carthamone & 51.03 & 0.2 & Carthami Flos \\
\hline MOL002719 & 6-Hydroxynaringenin & 33.23 & 0.24 & Carthami Flos \\
\hline MOL002721 & quercetagetin & 45.01 & 0.31 & Carthami Flos \\
\hline MOL002757 & 7,8-dimethyl-1H-pyrimido[5,6-g]quinoxaline-2,4-dione & 45.75 & 0.19 & Carthami Flos \\
\hline MOL002773 & beta-carotene & 37.18 & 0.58 & Carthami Flos \\
\hline MOL000358 & beta-sitosterol & 36.91 & 0.75 & Carthami Flos \\
\hline MOL000422 & kaempferol & 41.88 & 0.24 & Carthami Flos \\
\hline MOL000449 & Stigmasterol & 43.83 & 0.76 & Carthami Flos \\
\hline MOL000953 & CLR & 37.87 & 0.68 & Carthami Flos \\
\hline MOL000098 & quercetin & 46.43 & 0.28 & Carthami Flos \\
\hline
\end{tabular}


Figures

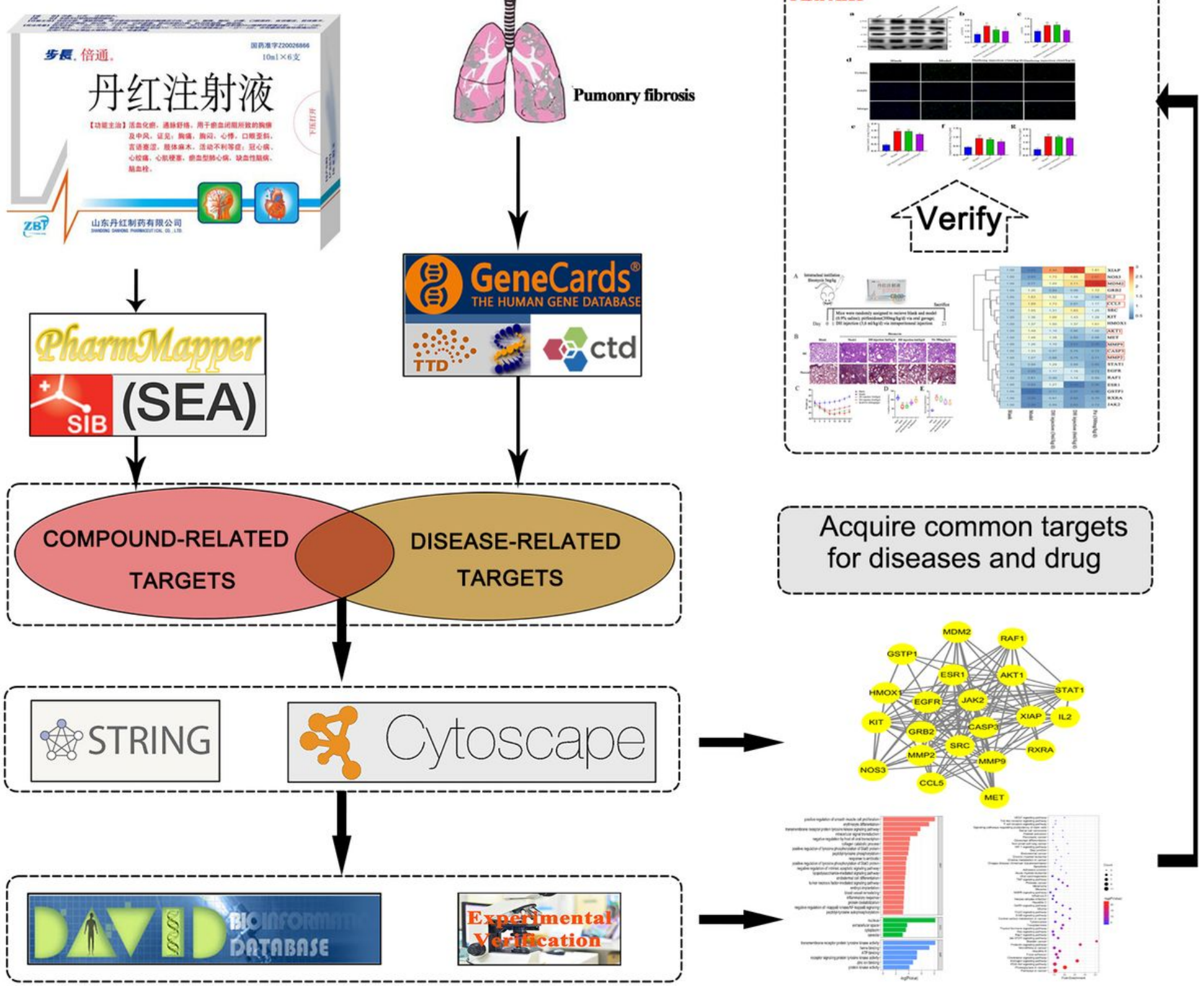

Figure 1

Technological road-map 


\section{Danshen}

\section{Honghua}

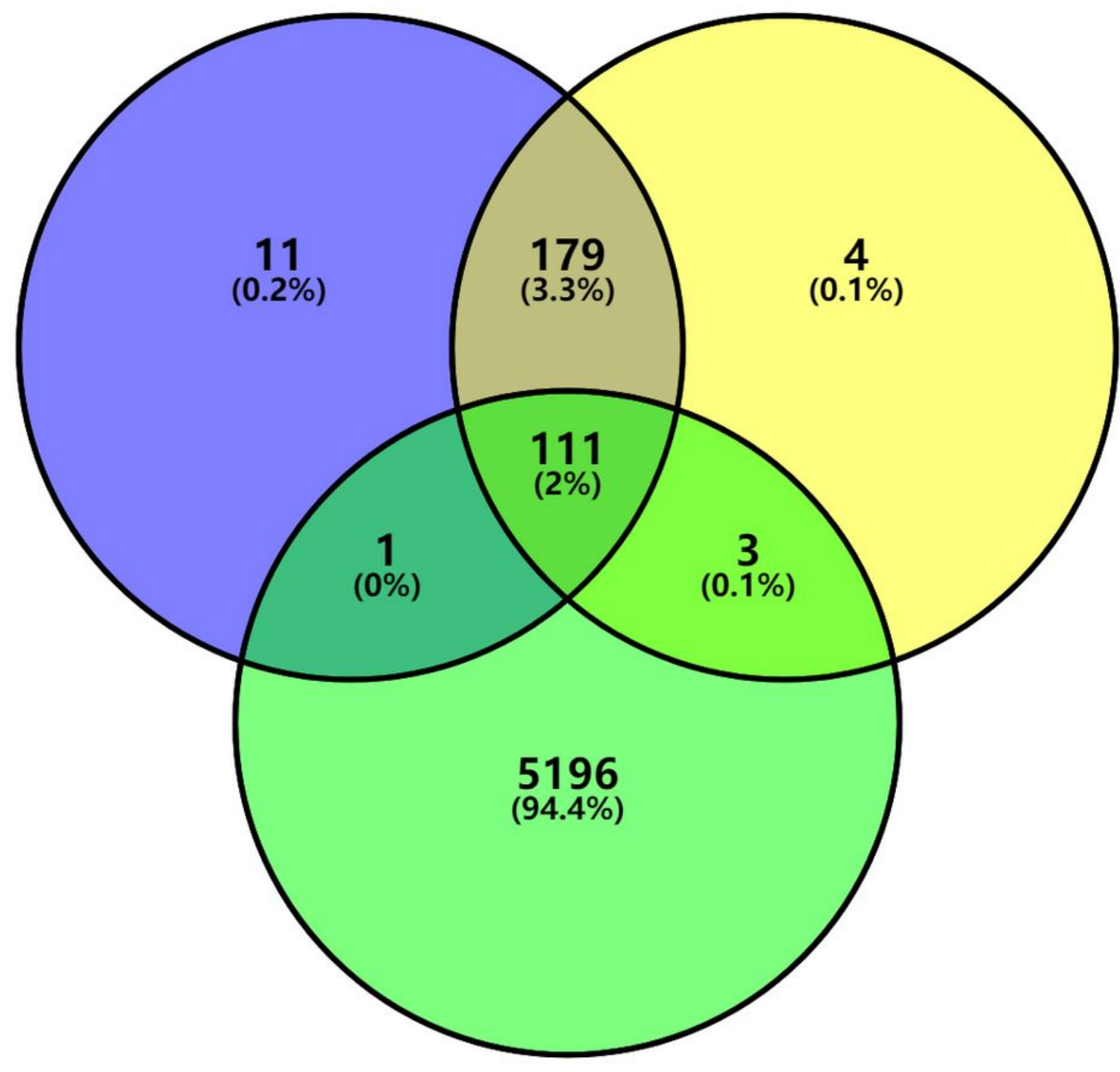

Pulmomary fibrosis

Figure 2

Drug-disease intersection targets 


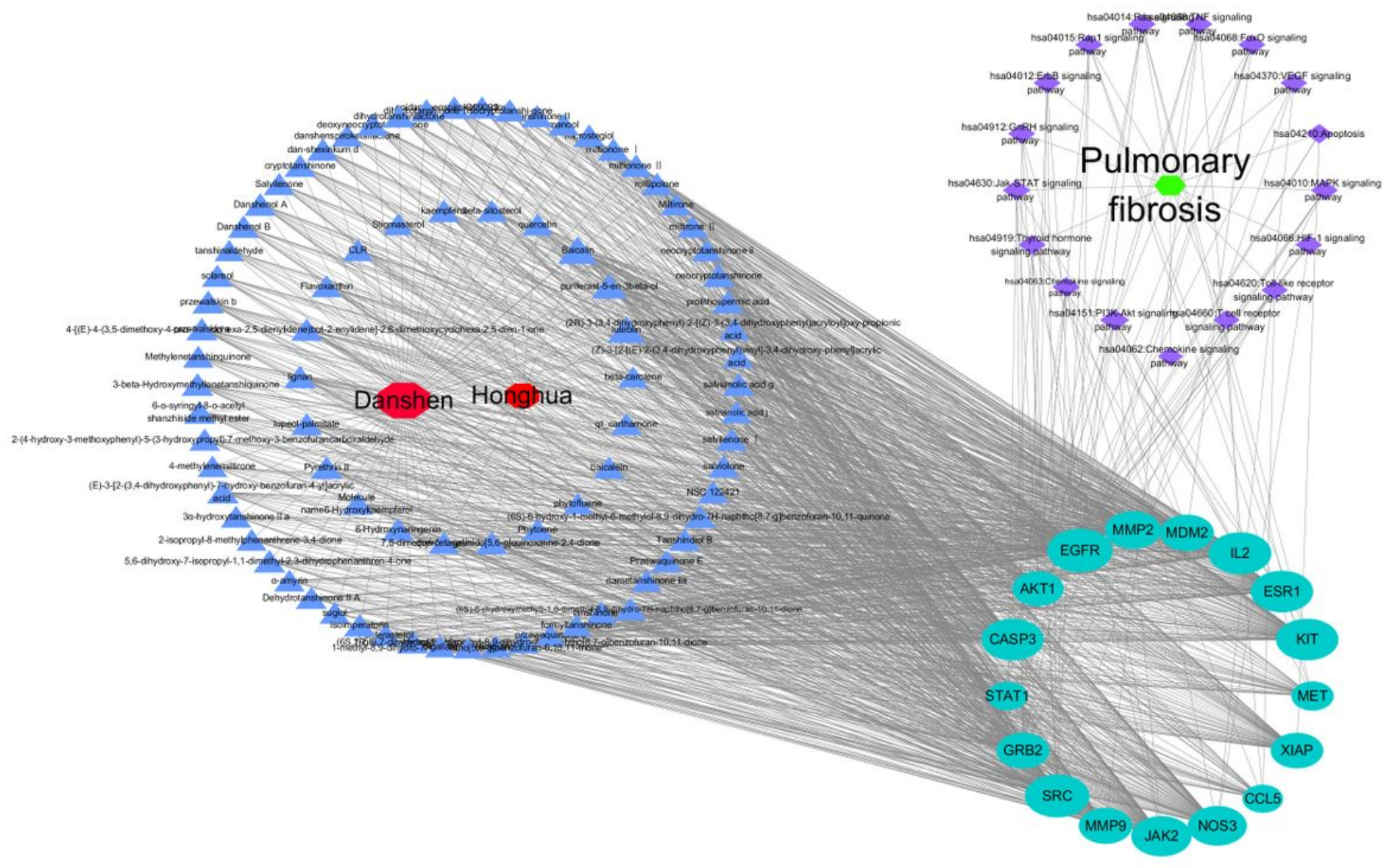

Figure 3

Anti-PF network diagram of the active ingredients of Danhong injection

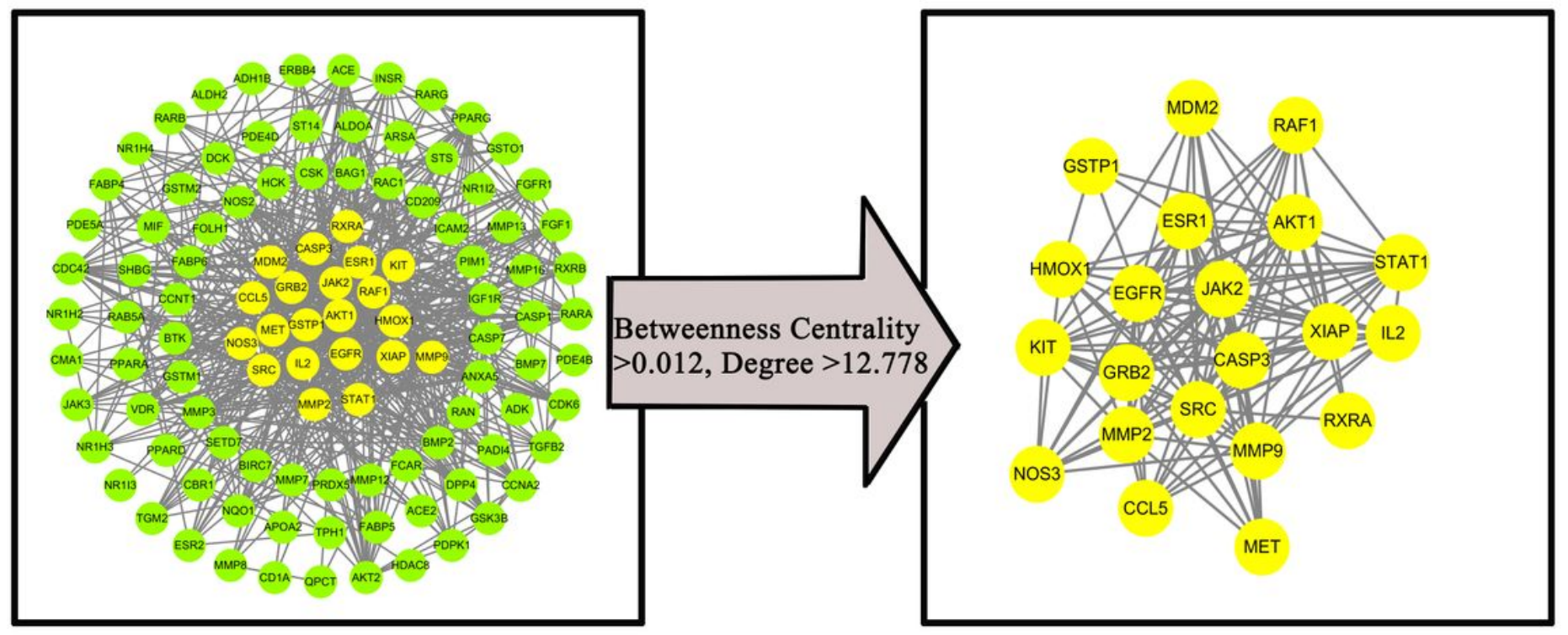

Figure 4

Screening of the core targets of the active ingredient of Danhong injection against PF 
a

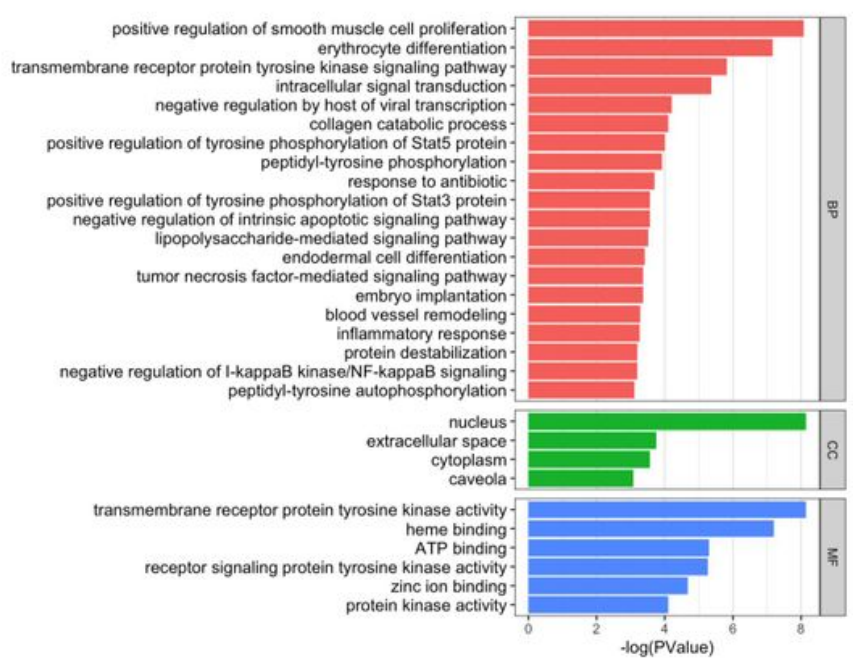

b

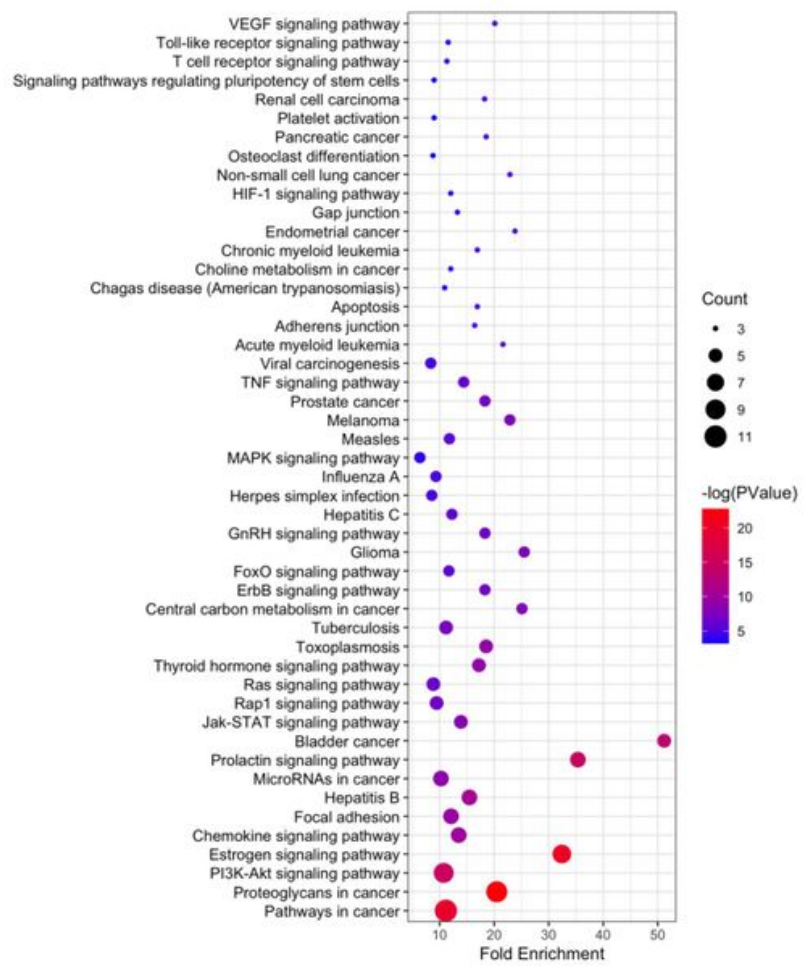

Figure 5

Anti-PF enrichment analysis of the active ingredients of Danhong injection. a GO enrichment analysis; $b$ KEGG enrichment analysis 
a Intratracheal instillation

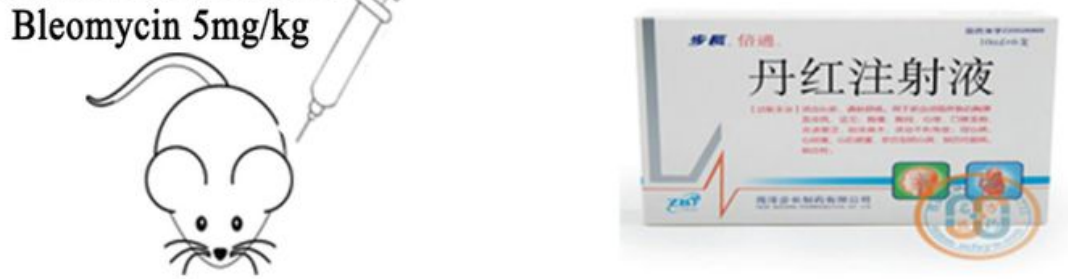

Sacrifice

Mice were randomly assigned to recieve blank and model ( $0.9 \%$ saline); pirfenidone $(300 \mathrm{mg} / \mathrm{kg} / \mathrm{d})$ via oral gavage;

Day $01 \mathrm{DH}$ injection $(3,6 \mathrm{ml} / \mathrm{kg} / \mathrm{d})$ via intraperitoneal injection

b

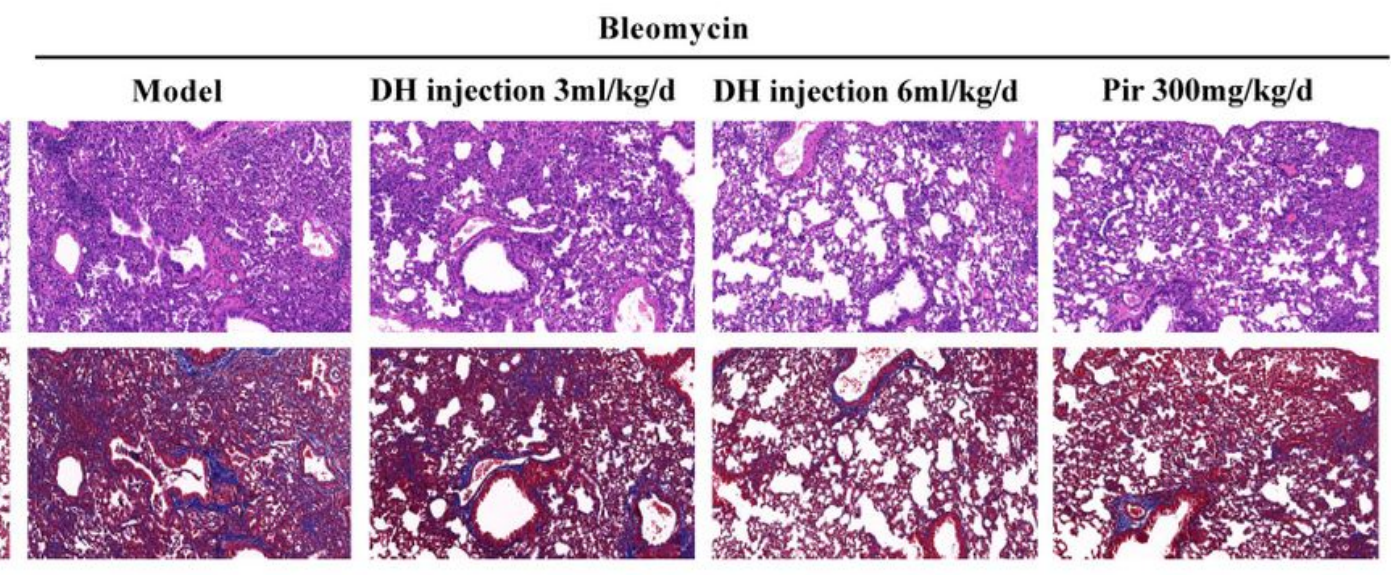

c

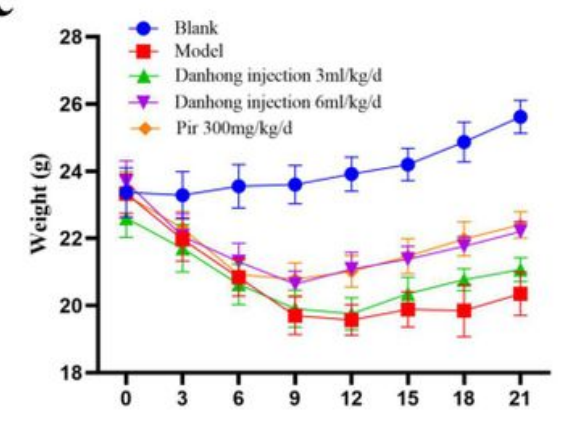

d

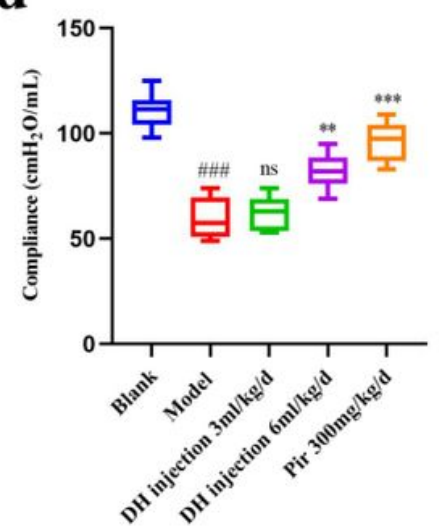

e

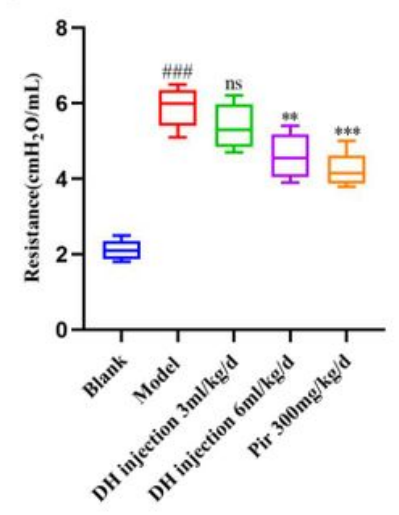

Figure 6

Danhong injection attenuates bleomycin-induced PF in mice. a Modeling diagram. b. Image of H\&E staining and Masson staining obtained at 20xmagnifcation in the lung. c Body weight. $d$ Lung compliance. e Lung resistance. All of the data are expressed as mean $\pm S D(n=6)$, \#\#\#P<0.01 compared with blank group ${ }^{*} \mathrm{P}<0.05$ compared with model group, ${ }^{\star *} \mathrm{P}<0.01$ compared with model group, ${ }^{* *} \mathrm{P}<0.001$ compared with model group and $\mathrm{ns}=$ no significant compared with model group. Scale bar $=50 \mu \mathrm{m}$ 
$\mathbf{a}$

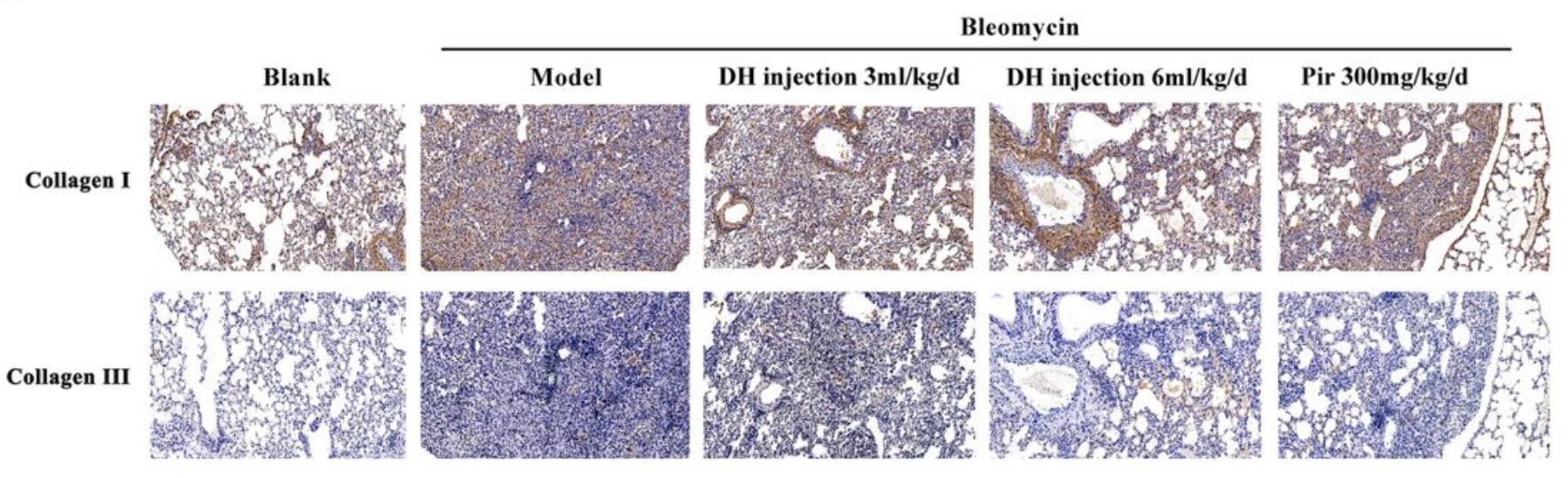

b

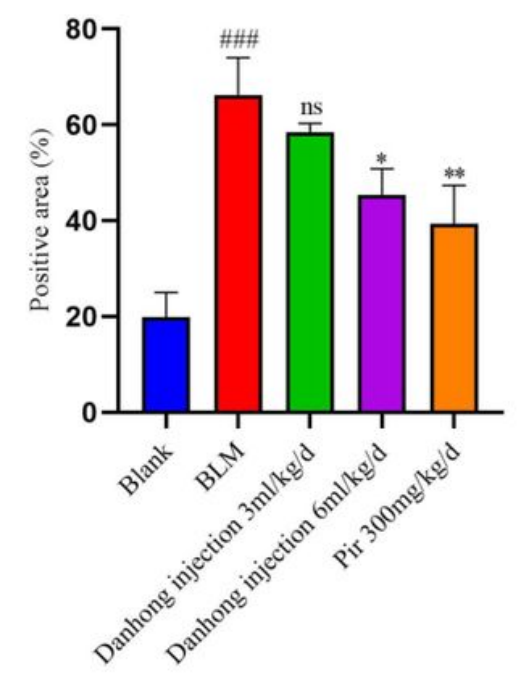

c

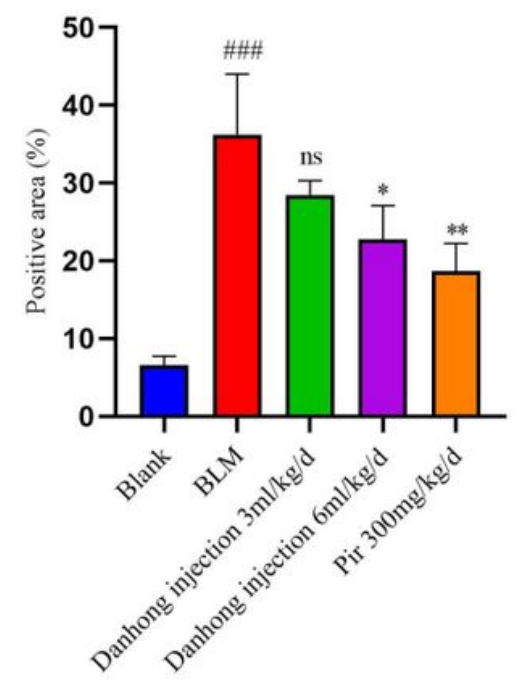

d

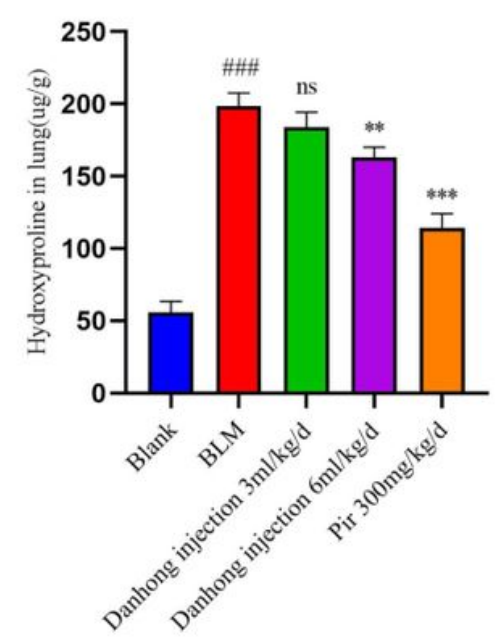

\section{Figure 7}

Dan Hong Injection reduced collagen deposition in the lungs of mice with bleomycin-induced PF. a. immunohistochemistry staining of Collagen I and collagen III in lung and image of immunohistochemistry obtained at 20xmagnifcation in lung. b, c Histology score of the pulmonary fibrosis calculated by Collagen I and collagen III. $d$ Contents of Hydroxyproline in lung tissue. All of the data are expressed as mean $\pm S D(n=6)$, \#\#\#P<0.001 compared with blank group $* P<0.5$ compared with model group, ${ }^{\star *} \mathrm{P}<0.01$ compared with model group, ${ }^{\star \star \star} \mathrm{P}<0.001$ compared with model group and $\mathrm{ns}=$ no significant compared with model group. Scale bar $=50 \mu \mathrm{m}$ 


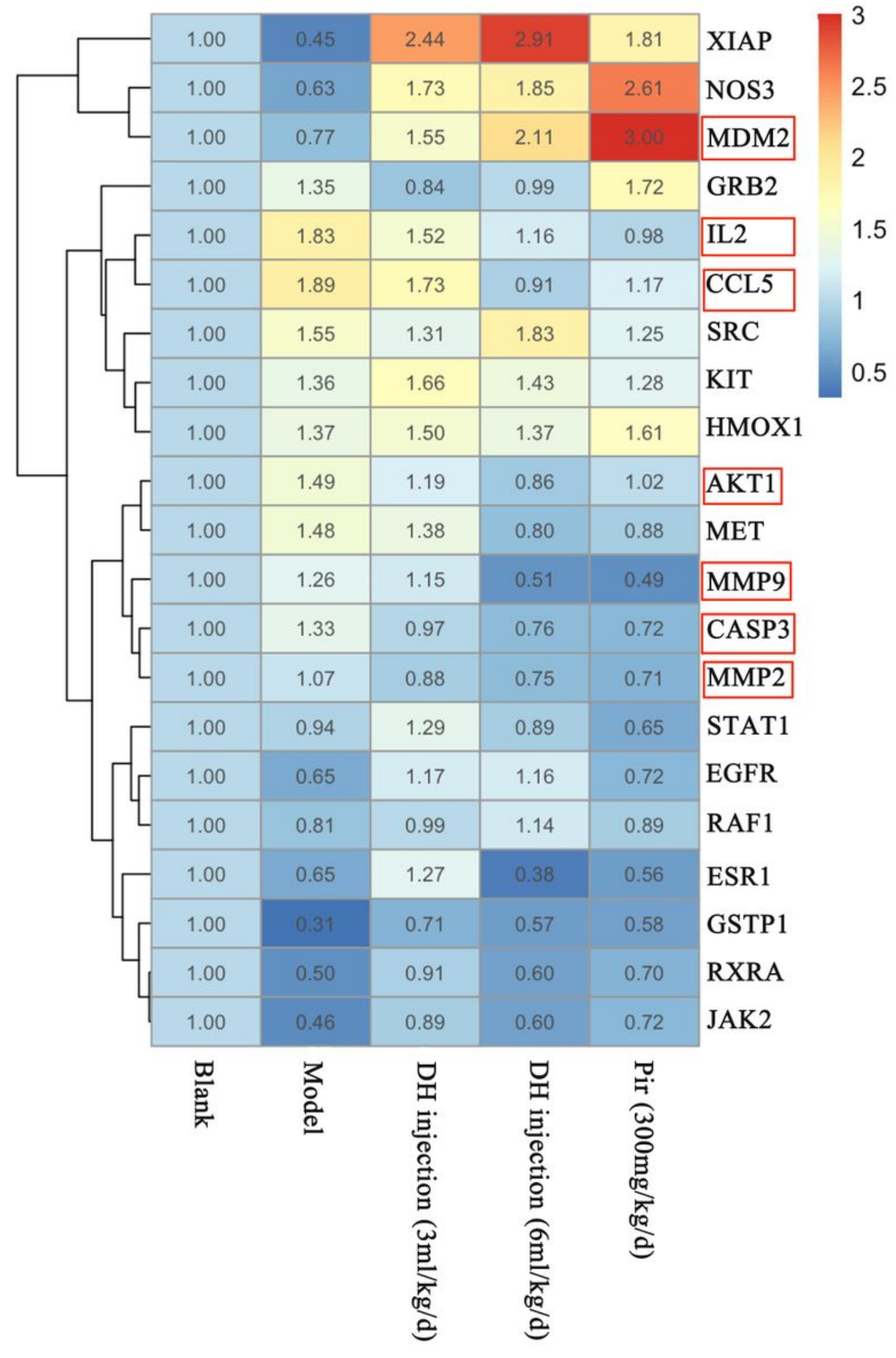

\section{Figure 8}

Effect of Danhong injection on the transcription of key target genes in lung tissue of mice with PF. Realtime qPCR analysis of genes, relative expression of XIAP, NOS3, MDM2, GRB2, IL-2, CCL5, SRC, KIT, HMOX1, AKT1, MET, MMP9, CASP3, MMP2, STAT1, EGFR, RAF1, ESR1, GSTP1, RXRA, JAK2 and make a heatmap. All of the data are expressed as mean $\pm S D(n=3)$. 

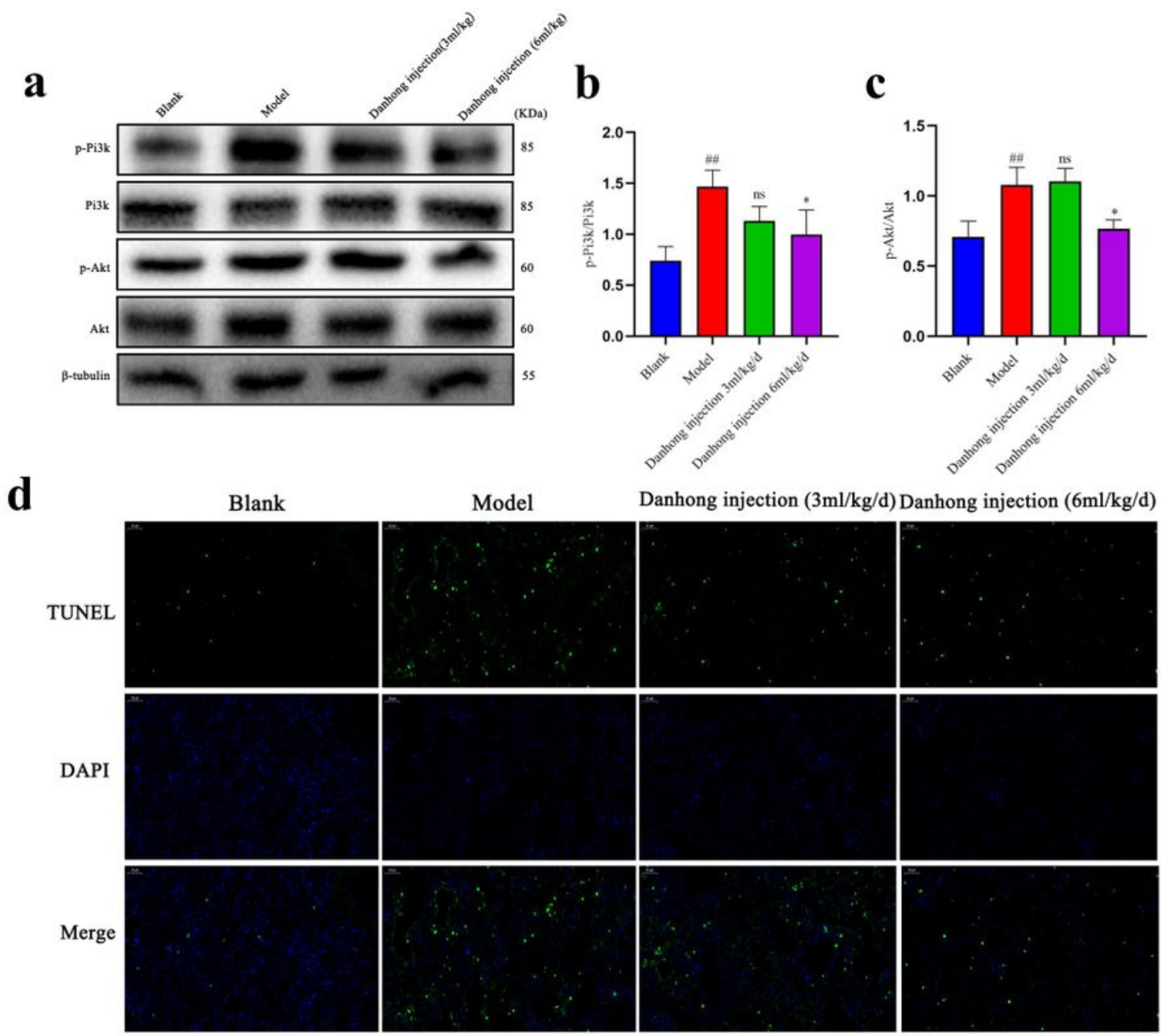

$\mathbf{e}$

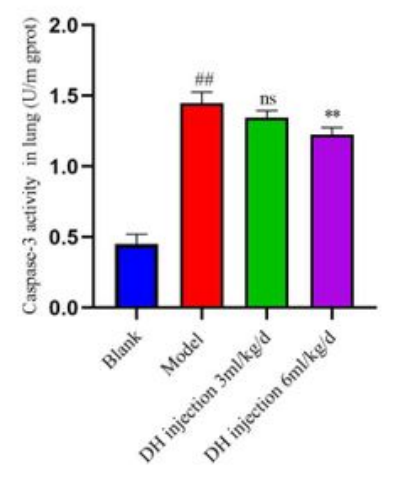

f

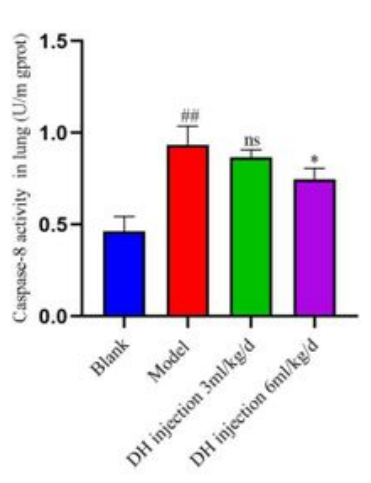

g

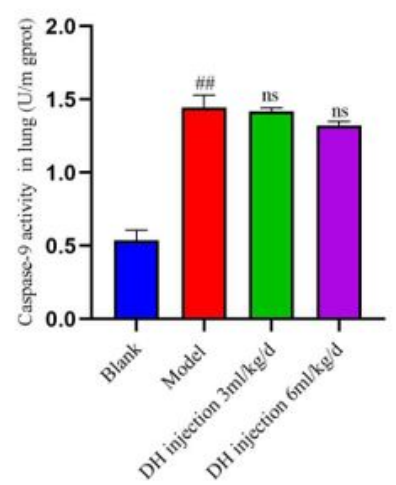

\section{Figure 9}

Danhong injection exerts antifibrotic effects via Pi3k/Akt signaling pathway. a b, c Western blotting of p$\mathrm{Pi} 3 \mathrm{k}, \mathrm{Pi} 3 \mathrm{k}, \mathrm{p}$-Akt, Akt, and $\beta$-tubulin protein in lung. $\mathrm{d}$ Immunofluorescence staining of apoptotic cell. DAPI was used to stain the nucleus. $d, e, f$ Contents of Caspase -3. Caspase-8DCaspase-9 in lung tissue. All of the data are expressed as mean $\pm S D(n=3)$, \#\#P<0.01 compared with blank group * $P<0.05$ compared 
with model group ${ }^{*} \mathrm{P}<0.01$ compared with model group and $\mathrm{ns}=$ no significant compared with model group. Scale bar $=50 \mu \mathrm{m}$ 